\title{
Density Gradient Ultracentrifugation of Nanotubes: Interplay of Bundling and Surfactants Encapsulation
}

\author{
F. Bonaccorso, ${ }^{\dagger}$ T. Hasan,${ }^{\dagger}$ P. H. Tan, ${ }^{\dagger,}$ C. Sciascia, ${ }^{\dagger}$ G. Privitera,${ }^{\dagger}$ G. Di Marco, ${ }^{\S}$ \\ P. G. Gucciardi, ${ }^{\S}$ and A. C. Ferrari*, \\ Engineering Department, Cambridge University, Cambridge CB3 OFA, United Kingdom, State Key Laboratory \\ for Superlattices and Microstructures, Institute of Semiconductors, Beijing 100083, China, and CNR-Istituto per \\ i Processi Chimico-Fisici, Viale F. Stagno D’Alcontres 37, 98158 Messina, Italy
}

Received: April 4, 2010; Revised Manuscript Received: July 21, 2010

\begin{abstract}
Density gradient ultracentrifugation (DGU) has emerged as a promising tool to prepare chirality enriched nanotube samples. Here, we assess the performance of different surfactants for DGU. Bile salts (e.g., sodium cholate (SC), sodium deoxycholate (SDC), and sodium taurodeoxycholate (TDC)) are more effective in individualizing Single Wall Carbon Nanotubes (SWNTs) compared to linear chain surfactants (e.g., sodium dodecylbenzene sulfonate (SDBS) and sodium dodecylsulfate (SDS)) and better suited for DGU. Using SC, a narrower diameter distribution $(0.69-0.81 \mathrm{~nm})$ is achieved through a single DGU step on CoMoCAT tubes, when compared to SDC and TDC $(0.69-0.89 \mathrm{~nm})$. No selectivity is obtained using SDBS, due to its ineffectiveness in debundling. We assign the reduced selectivity of dihydroxy bile salts (SDC and TDC) in comparison with trihydroxy SC to the formation of secondary micelles. This is determined by the number and position of hydroxyl $(-\mathrm{OH})$ groups on the $\alpha$-side of the steroid backbone. We also enrich CoMoCAT SWNTs in the 0.84-0.92 nm range using the Pluronic F98 triblock copolymer. Mixtures of bile salts (SC) and linear chain surfactants (SDS) are used to enrich metallic and semiconducting laser-ablation grown SWNTs. We demonstrate enrichment of a single chirality, $(6,5)$, combining diameter and metallic versus semiconducting separation on CoMoCAT samples.
\end{abstract}

\section{Introduction}

One of the main goals in nanotube research is to grow ondemand tubes of well-defined chirality. However, to date most approaches result in heterogeneous samples, with a limited success in the selective growth of semiconducting $(\mathrm{s}),{ }^{1-4}$ metallic $(\mathrm{m}),{ }^{5,6}$ or narrow chirality distribution ${ }^{7,8}$ single wall carbon nanotubes (SWNTs).

On one hand, the separation of SWNTs in all the chiralities originally present in the raw material, or the growth of chirality controlled samples, would be the ideal way to exploit their full potential in any applications, since one could mix the different chiralities according to the needs. Single chirality tubes would be ideal for the reproducible and large scale realization of many SWNTs-based devices, such as light-emitters, detectors, and interconnects, and optimized optical devices for single wavelength operation. Although huge progresses were made, we are still far from this ideal scenario.

On the other hand, several applications do not require single chirality but a lesser degree of selectivity. For example the use of SWNTs as saturable absorbers ${ }^{9-15}$ suffices diameter selection and benefits from the presence of small bundles. ${ }^{9-12}$ When tubes are used as filters, ${ }^{16}$ only material above or below a certain diameter is required. ${ }^{16} \mathrm{~m} / \mathrm{s}$ separation is fundamental for applications such as transparent conductors. ${ }^{17}$ In some applications, m- or s-SWNTs of well-defined diameters are needed. For example, by tuning the diameter distribution of sorted m-SWNTs

* To whom correspondence should be addressed. Telephone: +441223 7 48351. Fax: +44 12237 48348. E-mail: acf26@eng.cam.ac.uk.

Cambridge University.

* Institute of Semiconductors

${ }^{\S}$ CNR-Istituto per i Processi Chimico-Fisici. it is possible to fabricate transparent conductive films with tunable optical transmittance from the visible to the nearinfrared. ${ }^{17}$ Bundles of targeted composition are also interesting. $\mathrm{m} / \mathrm{s}$ separation can be used to control the relaxation of the excited states of s-SWNTs in bundles (by adjusting the relative $\mathrm{m} / \mathrm{s}$ population), hence, their photonics performance. ${ }^{9-14}$ Bundles can be used in optical trapping experiments, ${ }^{18-20}$ to study light induced rotations, ${ }^{19}$ to assemble photonic force microscopes of unprecedented resolution. ${ }^{20}$ Bundles dispersed in liquid crystals ${ }^{21}$ may form well-aligned systems leading to large nonlinear optical effects ${ }^{22,23}$ with enhanced electro-optical properties. ${ }^{24,25}$ Bundles could be engineered to exploit exciton energy transfer (EET) ${ }^{26,27}$ between donor (larger bandgap) and acceptor (smaller bandgap) tubes to realize excitonic antennas. In photovoltaic devices absorption over a wide wavelength range is important to boost efficiency. ${ }^{28} \mathrm{EET}$ in a bundle of tailored composition can extend the absorption range leading to enhanced quantum efficiency with a wide photoresponse from near UV through visible and near-infrared.

Different approaches for postgrowth selection have been proposed in literature. Selection can be achieved by dispersion and individualization in solution, followed by a sorting process. ${ }^{29-56}$ Debundling can be achieved by ultrasonication, with or without surfactants..$^{57-72}$ Depending on the procedure and/or surfactants/ polymers, tubes can be sorted by length, ${ }^{29-32}$ diameter, ${ }^{31-41}$ chirality, ${ }^{52-56,73} \mathrm{~m} / \mathrm{s}$ nature, ${ }^{33,37,42-51}$ and handedness. ${ }^{74-77}$

One strategy relies on the "amplification" of their density differences by covalent ${ }^{78-81}$ or noncovalent ${ }^{65,82,83}$ functionalization. Established procedures for the separation of molecules, such as size-exclusion or ion-exchange chromatography, ${ }^{84,85}$ electrophoresis, ${ }^{86,87}$ and conventional or density gradient ultracentrifugation (DGU) ${ }^{88-90}$ can then be used. ${ }^{29-56}$ Each method 
has advantages and disadvantages. Sorting techniques based on covalent functionalization, for example, alter the electronic structure, even after functional groups removal, ${ }^{78,79,91}$ since the sidewall attachment of reactive groups disrupts the $\pi$-network. ${ }^{92,93}$ Diameter or $\mathrm{m} / \mathrm{s}$ separation is possible by chromatography, through wrapping with single strand DNA (ss-DNA). ${ }^{33,34,53,65}$ Reference 56 identified 25 sequences, through a painstaking search of a $10^{60}$ DNA library, enabling chromatographic purification of up to $90 \%$ of 12 single-chirality s-SWNTs. However, DNA has lower selectivity for m-separation ${ }^{94}$ and is difficult to handle and remove. . $^{33,34,65}$

In gel, ${ }^{31,32,49}$ capillary, ${ }^{35}$ or free-solution electrophoresis, ${ }^{46}$ separation is based on the electrophoretic mobility of SWNTs under a direct $(\mathrm{dc})$ or alternating current (ac) field. ${ }^{31,32,35,46,49}$ Gel electrophoresis, ${ }^{31,32,49}$ in which SWNTs travel through a gel (e.g., agarose),${ }^{31,32,49}$ can sort tubes by length, ${ }^{31}$ diameter, ${ }^{31,32,34,49}$ and by their $\mathrm{m}$ - or s-nature. ${ }^{49}$ Capillary electrophoresis can separate by length, ${ }^{95}$ as well as bundled from isolated tubes, exploiting differences in electrophoretic mobility, which depends on tube diameter and bundle size. ${ }^{35}$ Free-solution electrophoresis was used to separate m- from s-SWNTs. ${ }^{46}$ Reference 42 showed that using an ac field (dielectrophoresis) m-SWNTs, more polarizable than s-SWNTs, can be deposited between the electrodes of an array. This was recently extended to graphene ${ }^{96}$ and used to assemble SWNT and graphene based electroluminescent devices. ${ }^{97}$ This method also enables sorting by diameter. $^{44}$ Reference 45 improved the process to yield $1 \mu \mathrm{g}$, compared to the $100 \mathrm{pg}$ of the first report. ${ }^{42}$ Reference 98 scaled up the procedure in microfluidic channels, reaching $\sim 70 \%$ m-SWNTs, when compared to the starting material. ${ }^{98} \mathrm{~m}-\mathrm{SWNTs}$ could be further separated by diameter via frequency tuning. ${ }^{98}$ Reference 47 reported separation of up to $70 \% \mathrm{~m}$ - and $95 \%$ s-SWNTs by a gel-squeezing technique, exploiting selective adsorption of s-SWNTs on agarose gel, whereas m-SWNTs remain encapsulated in sodium dodecylsulfate (SDS) micelles in the squeezed solution. Sorting by ultracentrifugation is also possible. ${ }^{50,91,99,100}$ However, it results in broad diameter and length distribution, unless preceded by selective chemical treatment..$^{50,99,100}$

Reference 36 demonstrated DGU as a promising technique to separate SWNTs by diameter. In DGU, aqueous dispersions of surfactant-encapsulated tubes are ultracentrifuged in a preformed density gradient medium (DGM). During the process, they move along an ultracentrifuge cell, dragged by the centrifugal force, until they reach the corresponding isopycnic points, i.e. the point where their buoyant density equals that of the surrounding DGM. ${ }^{101}$ Such process depends only on the buoyant density of the particles (i.e., nanotubes in this instance) and is also called isopycnic separation. ${ }^{88}$ The buoyant density of a nanotube-surfactant complex is defined as the density of the medium at the corresponding nanotube isopycnic point and is measured in $\mathrm{g} / \mathrm{cm}^{3}$. It depends on the local composition of the dispersion, the type of surfactant and may be different for different $\mathrm{DGM}^{102}$ or $\mathrm{pH}$ values. ${ }^{102}$ Sorting is achieved by exploiting the buoyant density difference of surfactant encapsulated tubes. ${ }^{36,37,39,41}$ This results in a spatial separation inside an ultracentrifuge cell, overcoming the limitations of conventional ultracentrifugation in a density constant medium. ${ }^{41}$ Reference 37 first reported the separation between $(6,5)$ and $(7,5)$. These have a diameter difference of only $0.7 \AA .{ }^{103}$ Moreover, ref 37 obtained dispersions of highly enriched $(6,5)$ (>83\% among s-SWNTs) after three DGU iterations. Reference 76 separated left from right handed SWNTs of the same $(6,5)$ chirality by encapsulating them with the chiral surfactant Sodium
Cholate (SC), and exploiting the changes in buoyant density determined by small variations in the ordering of SC around left and right-handed SWNTs. Recently, ref 77 improved the process, separating seven pairs of enantiomers using a mixture of surfactants (SC and SDS). DGU is a more flexible technique compared to other methods, such as chromatography and electrophoresis, as it is compatible with SWNTs from different sources and works in noncovalent and reversible chemistry. ${ }^{37,91}$ Moreover, it can be used iteratively. ${ }^{37}$ Scale-up can be achieved with ultracentrifuges used in the pharmaceutical industry. ${ }^{91}$

DGU allows $\mathrm{m} / \mathrm{s}$ separation with minimal modification of the protocol used for diameter separation. ${ }^{37}$ Reference 37 first reported $\mathrm{m} / \mathrm{s}$ separation of laser ablation (LA) SWNTs using a cosurfactant mixture (SC + SDS). Reference 48 separated m-SWNTs from three different sources (LA, HiPco and CoMoCAT). Reference 104 achieved length separation exploiting the transient nanotube motion in response to an applied centripetal acceleration field.

Even though DGU is now extensively used, a detailed understanding of the sorting mechanism is still lacking. Natural bile salts ${ }^{37}$ seem effective for diameter separation, in contrast to linear chain surfactants, such as Sodium Dodecylbenzene Sulfonate (SDBS) and SDS. However, to date only SDS among linear chain surfactants was used to separate m- from s-SWNTs, in combination with $\mathrm{SC},{ }^{37}$ or cosurfactant mixtures of SC-SDC (sodium deoxycholate). ${ }^{48}$ It remains unclear why the separation is so strongly dependent on the surfactant or on the composition of the cosurfactant mixture.

A successful DGU sorting mainly depends on the following:

(1) Nanotube individualization (debundling) in the starting dispersions (Figure 1a). Differences in buoyant density can be correlated to specific tubes only if individualized tubes are encapsulated by the surfactant micelles.

(2) Uniform sidewall coverage (Figure 1b). This is ruled by the adsorption of surfactant molecules, and their aggregation, which can lead to clusters formation (i.e., aggregates of several molecules around the tube sidewalls).

Table 1 summarizes the current state of the art of SWNT sorting by different techniques, DGU in particular. In this paper, we will use "sorting" to indicate a generic process of postgrowth nanotube selection. We use "separation" to indicate a process resulting in a sample with diameter in a certain range or to indicate $\mathrm{m} / \mathrm{s}$ sorting. We use "enrichment" to indicate a process resulting in an increase of the percentage of nanotubes with certain chirality with respect to the pristine material.

We thus classify the DGU in

1 diameter separation

1a range (between two different diameters)

$1 \mathrm{~b}$ threshold (above or below a certain diameter)

2 metallic versus semiconducting separation

3 diameter sorting of $\mathrm{m} / \mathrm{s}$ separated samples

4 single chirality enrichment

5 length separation

6 enantiomer separation.

In this paper we cover 1a (sections 2.3 .3 and 3.2), 2, 3 (sections 2.3.4 and 3.3), and 4 (sections 2.3.5 and 3.4). We do not consider enantiomer, length, and threshold diameter separation because they require the investigation of other classes of surfactant/polymer (cases $1 \mathrm{~b}$ and 6) or a different experimental procedure (case 5).

\section{Experimental Section}

2.1. Pristine Nanotubes. We use CoMoCAT ${ }^{105,106}$ (South West Nanotechnologies; batch: CAU-A002) and LA SWNTs. ${ }^{107,108}$ 

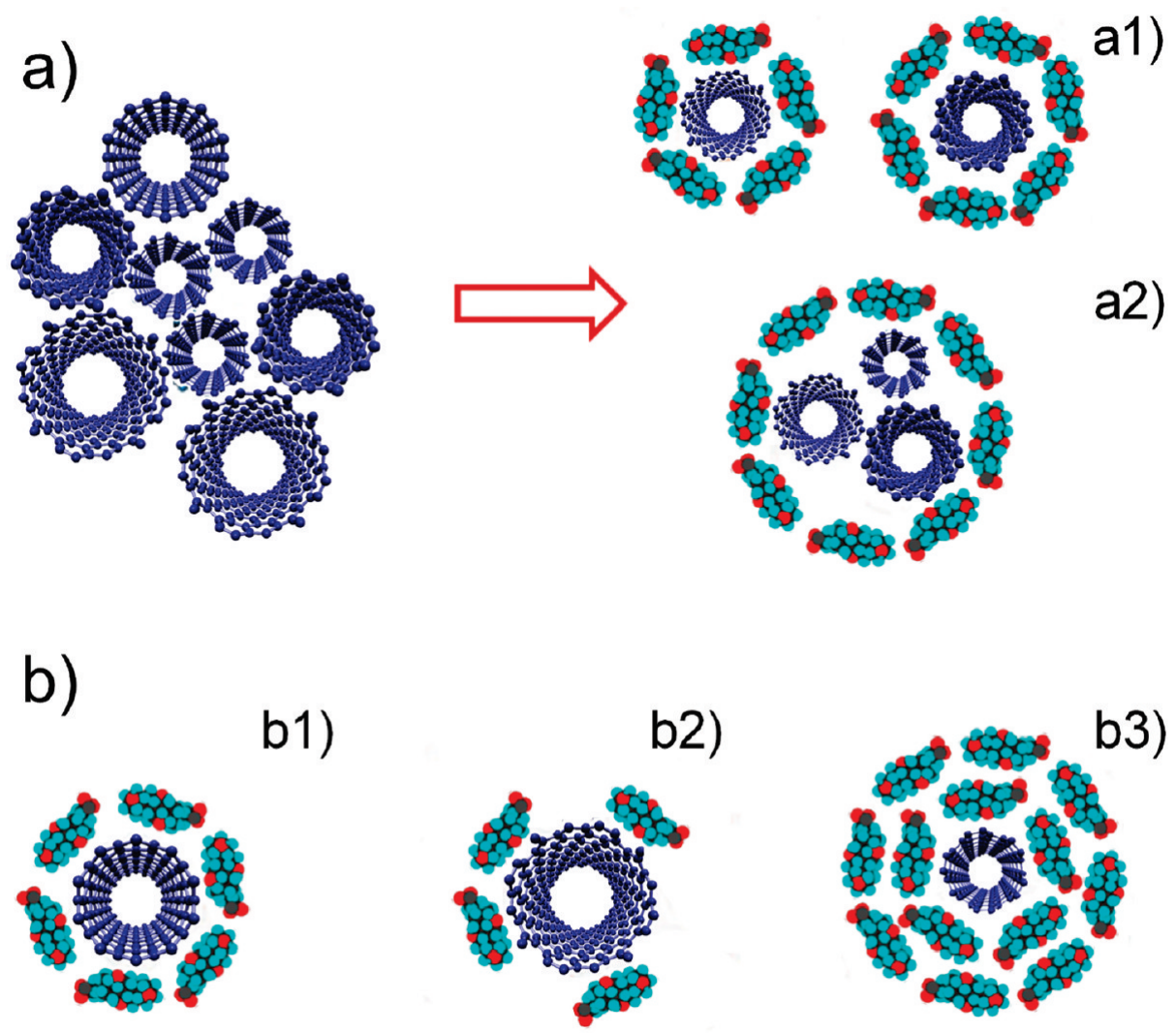

Figure 1. Schematic representation of (a) debundling and adsorption of surfactant on SWNT sidewalls; (b) surfactant coverage and clustering. Successful DGU sorting requires both (a1) individualization and (b1) uniform sidewall surfactant coverage. On the contrary, (a2) bundling, (b2) nonuniform coverage, and (b3) cluster formation, hinder the sorting process.

TABLE 1: Different Strategies to Sort SWNTs ${ }^{a}$

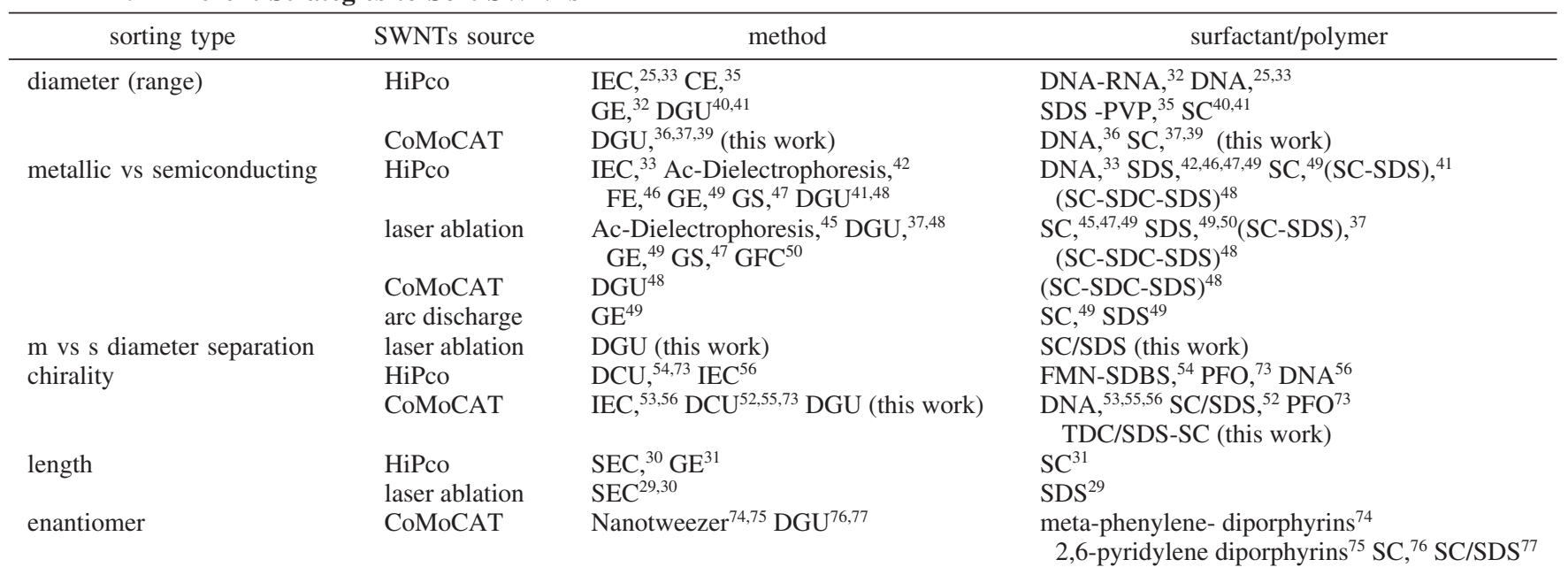

${ }^{a} \mathrm{SEC}=$ size exclusion chromatography; GE = gel electrophoresis; IEC = ion exchange chromatography; CE = capillary electrophoresis; DGU = density gradient ultracentrifugation; DCU = density constant ultracentrifugation; FE = free solution electrophoresis; GS = gel-based filtration; GFC = gel filtration chromatography; DNA = deoxyribonucleic acid; RNA = ribonucleic acid; PFO = poly $(9,9$-dioctylfluorenyl-2,7-diyl); FMN = flavin mononucleotide.

CoMoCAT are ideally suited for diameter separation and single chirality enrichment, since they have a narrow diameter distribution to start with $(0.6-1.2 \mathrm{~nm}) .{ }^{105}$ We use LA-SWNTs with a diameter distribution $0.9-1.6 \mathrm{~nm}$, and an average diameter $1.2-1.3 \mathrm{~nm},{ }^{107,108}$ since this range provides a bandgap suitable for telecommunications. ${ }^{9-14}$ However, the DGU procedures presented here are also applicable for diameter distributions different from the ones we used.

2.2. Dispersion and Individualization of SWNTs in Aqueous Solutions. An important prerequisite for SWNTs sorting is their individualization. Therefore, we first evaluate the performances of six surfactants, among the most widely used in literature, ${ }^{58-60,109}$ for the encapsulation of individualized (CoMoCAT) SWNTs. We consider linear chain surfactants (SDS and SDBS), bile salts (SDC, TDC, and SC), and a nonionic difunctional triblock (PEO-PPO-PEO) copolymer (Pluronic F98). This consists of 2 terminal hydroxyl groups PEO (poly(ethylene oxide)) and a central PPO (poly(propylene oxide)) chain. ${ }^{110}$ The chemical structure is schematized in Figure 2.

SDS and SDBS have a flexible cylindrical body and form spherical or ellipsoidal micelles. ${ }^{11-114}$ In contrast, bile salts are 
<smiles>[Y10]=[Y10]=[Y10]</smiles><smiles></smiles><smiles></smiles>

SDBS; m.w. $\sim 348.48$<smiles>CC(C)(C)CCOS(=O)(=O)[O-]</smiles>

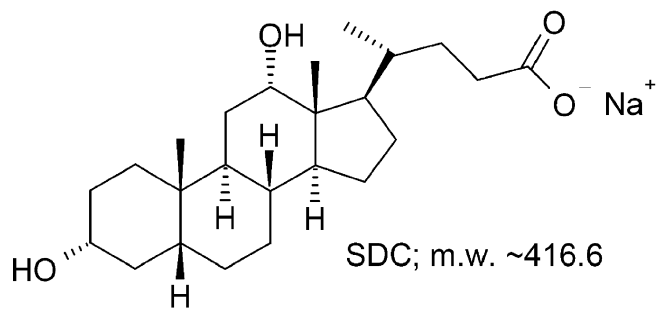

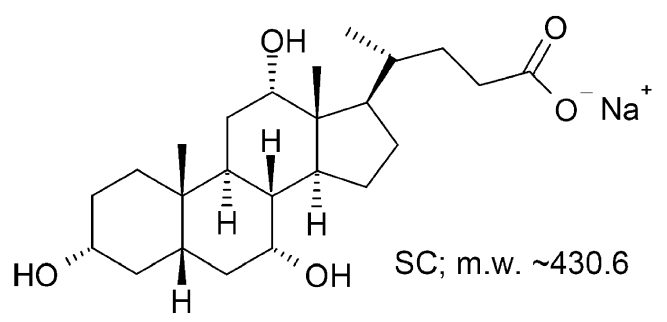

Figure 2. Structure and molecular weight of the surfactants used in this paper (adapted from refs 58 and 109).

rigid, consisting of a cholesterol group with dissimilar sides. ${ }^{115,116}$ Bile salts usually have a steroid skeleton with a carboxylic acid side-chain and one to three hydroxyl $(-\mathrm{OH})$ groups on the steroid backbone. ${ }^{115,116}$ They are amphiphilic, having both hydrophobic and hydrophilic sides. ${ }^{115,116}$

The six surfactants are used to prepare aqueous dispersions of CoMoCAT SWNTs. ${ }^{105}$ Approximately $0.2 \mathrm{mg} / \mathrm{mL}$ SWNTs are added to $10 \mathrm{~mL}$ of deionized (DI) $\mathrm{H}_{2} \mathrm{O}$ with $2 \% \mathrm{w} / \mathrm{v}$ surfactant, and then treated at $15^{\circ} \mathrm{C}$ in a $200 \mathrm{~W}, 20 \mathrm{kHz}$ sonicator bath (Nanoruptor, Diagenode) for $90 \mathrm{~min}$. Large bundles, together with insoluble materials, are removed via ultracentrifugation using an Optima Max-E ultracentrifuge (BeckmanCoulter) at $50 \mathrm{kRPM}(\sim 173000 \mathrm{~g})$ for $2 \mathrm{~h}$, with a MLA-80 fixed angle rotor.

2.3. Density Gradient Ultracentrifugation. 2.3.1. Density Gradient Medium and Density Gradient. The DGM choice is fundamental. Salts (such as cesium chloride, lithium chloride, sodium chloride, etc.), sucrose, and Optiprep, i.e., $60 \% \mathrm{w} / \mathrm{v}$ iodixanol $\left(\mathrm{C}_{35} \mathrm{H}_{44} \mathrm{I}_{6} \mathrm{~N}_{6} \mathrm{O}_{5}\right)$ solution in water ${ }^{117}\left(\rho=1.32 \mathrm{~g} \mathrm{~cm}^{-3}\right)$, are usually exploited in isopycnic separation. ${ }^{118,119}$ Due to low viscosity, density gradients produced with salts are less stable with respect to those using sucrose and Optiprep. ${ }^{120}$ Moreover, salts induce strong aggregation on the hydrophobic solutes ${ }^{121,122}$ that may affect the sorting process. On the contrary, the percentage of sucrose used as DGM can have a significant impact on sorting. ${ }^{123}$ Sucrose has high viscosity, exponentially increasing at high concentrations. ${ }^{123}$ Thus, ref 50, which used sucrose, carried out the procedure at very high $g(\sim 402000)$ and reported the outcome to be strongly dependent on both surfactant concentration and temperature. Optiprep is better suited due to its higher viscosity, with respect to salts, and density tunability, with respect to sucrose. Moreover, Optiprep has an almost constant viscosity as a function of the density in the gradient. ${ }^{124}$ By diluting Optiprep, the density profile can be shaped in different ways: linear, nonlinear, or step. ${ }^{125-128}$

Step gradients, formed by stacking layers of different density, ${ }^{126}$ are most effective for the separation of molecules with large density differences. ${ }^{126}$ The sharp change in density at the interface of two different layers stops molecules with a density smaller than the denser layer and lets the larger density molecules pass through. ${ }^{125,126}$

In nonlinear gradients, the DGM density changes nonlinearly along the cell. The sample is usually mixed with a salt (e.g., $\mathrm{CsCl}$ ) solution, and the density gradient is established during centrifugation. ${ }^{127}$ Nonlinear gradients are best suited to sediment particles over the entire length of an ultracentrifuge cell. ${ }^{127}$ In principle, nonlinear gradients are the most sensitive, since a variety of depth-density profiles can be produced according to the density variation, enabling trapping of particles of different densities along the cell length. Recently, ref 77 exploited a nonlinear density gradient for the separation of mirror-image isomers (enantiomers) of seven different chiralities. However, often there is no advantage over a linear gradient in the final separation, since the particles take a long time to reach equilibrium. ${ }^{129}$

Linear gradients can sort materials with buoyant density differences as little as $\sim 0.01 \mathrm{~g} \mathrm{~cm}^{-3}, 89,128$ depending on the gradient steepness. They can therefore be used to sort isolated surfactant-micelle encapsulated SWNTs with very small buoyant density difference, such as $(6,5)\left(1.06 \mathrm{~g} / \mathrm{cm}^{-3}\right)$ and $(7,5)(1.08$ $\mathrm{g} / \mathrm{cm}^{-3}$ ) dispersed in SC. ${ }^{41}$

The gradient density and its variation are both key to sorting. ${ }^{37}$ The gradient must be shaped to minimize the distance tubes travel to the isopycnic points. ${ }^{37}$ When designing the gradient, one needs consider that during centrifugation the density profile redistributes as the DGM responds to the centrifugal force, resulting in a steeper gradient over time. ${ }^{89}$ The protocol we use for DGU is shown in Figure 3. We start with ultrasonication in a water-surfactant solution, Figure $3 \mathrm{a}$, followed by preultracentrifugation, Figure $3 b$. The supernatant is then extracted, Figure $3 \mathrm{c}$, and used for DGU. A step gradient is formed by stacking different layers of Optiprep with gradually decreasing density, Figure 3d. The nanotube dispersion is then placed between two of the layers or at the top. A linear gradient is then produced by diffusion, Figure $3 e .{ }^{39,130}$ We use a swinging bucket rotor, since this is better than a fixed angle one in maximizing the diffusion length, because it allows the cell to 

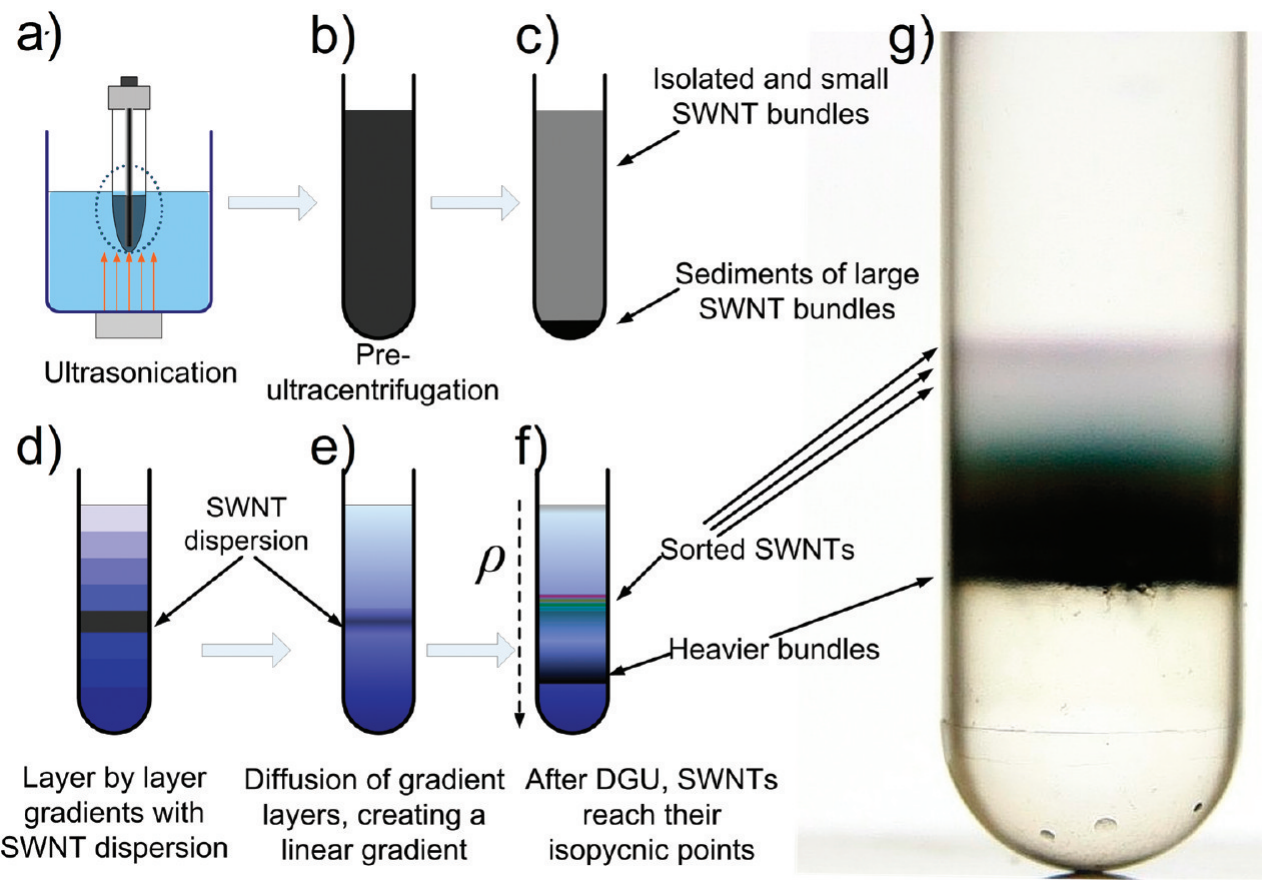

Figure 3. Schematic of SWNTs sorting by DGU. (a) Ultrasonication in an aqueous surfactant solution. (b) Preultracentrifugation. (c) Extraction of the supernatant fractions. (d) Formation of step gradient by placing four layers with decreasing Optiprep concentration on top of each other. ${ }^{37,39}$ The SWNT dispersion is inserted on top of the second layer. (e) Diffusion of the step gradient forms the linear density gradient. (f) SWNT sorting via DGU leads to SWNTs spatial separation in the ultracentrifuge cell. (g) Photograph of cell containing sorted SWNTs, with colored bands.

align orthogonally to the centrifugation axis, thus making sorting more effective.

The appearance of different color bands is an indication of SWNTs sorting, Figure $3 f^{37,39}$ The colors depend on the peak optical absorption. Thus, e.g., the purple color (non spectral redblue combination) of the top band in Figure $3 \mathrm{~g}$ is due to $(6,5)$ tubes that absorb at $\sim 570 \mathrm{~nm}\left(e h_{22}\right)$ (yellow). ${ }^{39}$

2.3.2. Fractionation. After DGU, the sorted SWNTs are removed, layer by layer, following the fractionation procedures developed in refs 37 and 39. Fractionation consists of extracting quantities of a mixture to a number of aliquots whose composition varies according to the density gradient of the original mixture. ${ }^{131}$ Fractionation methods are classified into three main categories: piston, ${ }^{132}$ down, ${ }^{133}$ and upward ${ }^{132}$ displacement. In the former, a piston, forced into the cell from above, displaces the gradient, while its tip collects liquid from the volume just below it. ${ }^{132}$ Downward displacement exploits an effluent, air ${ }^{134}$ or distilled water, ${ }^{135}$ inserted at the top of the cell, while the fractions are collected from the bottom, after piercing the cell with a needle. ${ }^{136}$ In upward displacement, a dense solution is inserted with a needle at the bottom pushing the gradient up into an inverted collection needle. ${ }^{132}$

We tested all three procedures for the fractionation of sorted SWNTs obtaining comparable results. For simplicity we selected upward displacement, using a syringe pump to extract small aliquots of sorted SWNTs. ${ }^{37,39}$ Fluorinert FC-40 $\left(\rho \approx 1.85 \mathrm{~g} / \mathrm{cm}^{3}\right.$; Sigma-Aldrich) is used as a high density chase solution. ${ }^{137}$ The extraction process is schematized in Figure 4. First, the distance between the top of the dispersion and the upper band is carefully measured using a slide caliper and the corresponding volume calculated. This is then extracted and discarded by injecting the same volume of Fluorinert at the bottom of the cell. Once the bands of interest are at the top, $\sim 70 \mu \mathrm{L}$ of Fluorinert is injected to extract the same volume of sorted SWNTs. The fractions are then diluted with $100 \mu \mathrm{L} 2 \%$ w/v surfactant aqueous solutions for optical characterization.

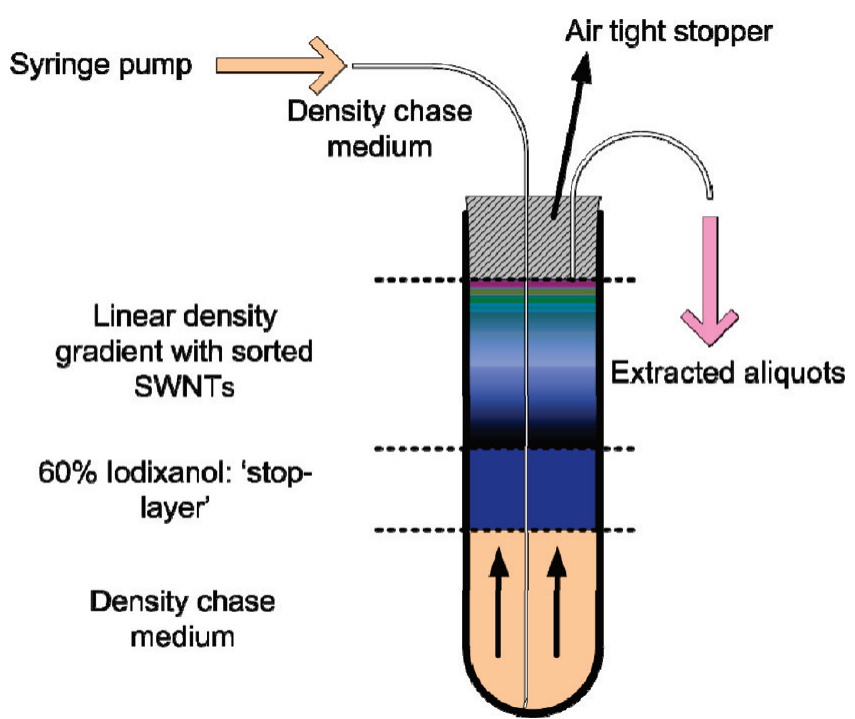

Figure 4. Schematic description of upward displacement fractionation. The colored bands illustrate the variation in density along the length of the ultracentrifuge cell. A small volume of density chase medium (typically $\sim 70-100 \mu \mathrm{L}$ ) is injected to extract the same volume of the dispersion of interest. ${ }^{37,39}$

2.3.3. Diameter Separation. Uniform surfactant coverage immediately links buoyant density to tube diameter, thus enabling an effective diameter separation by DGU.

The dispersion and individualization steps are carried out as for section 2.2. After ultrasonication, insoluble materials and large bundles are removed by 30-min ultracentrifugation at 54 kRPM (202 000g) in a Beckman Coulter MLA-80 fixed angle rotor at $15{ }^{\circ} \mathrm{C}$. The top $60 \%$ of this dispersion is decanted and used for DGU. The density gradient is formed in a Seton, ultra clear open-top, $14 \times 89 \mathrm{~mm}, 13.2 \mathrm{~mL}$ capacity cell by stacking different Optiprep layers with gradually decreasing density. At the bottom, we insert $1.5 \mathrm{~mL}$ of Optiprep stopping layer $(\rho \approx$ 
$1.32 \mathrm{~g} / \mathrm{cm}^{3}$ ) with an extra $2 \% \mathrm{w} / \mathrm{v}$ surfactant. We then insert $2.5 \mathrm{~mL}$ of Optiprep $50 \%$ (diluted with DI water $1: 4 \mathrm{v} / \mathrm{v}, \rho \approx$ $1.16 \mathrm{~g} / \mathrm{cm}^{3}$ ) added with $2 \% \mathrm{w} / \mathrm{v}$ surfactant. On top of this, we place $1.5 \mathrm{~mL}$ of SWNTs dispersion (density adjusted to 1.12 $\mathrm{g} / \mathrm{cm}^{3}$ by adding $100 \%$ Optiprep with $2 \%$ w/v surfactant). The cell is then filled with two more layers: $2.5 \mathrm{~mL}$ of Optiprep $25 \%$ (diluted with DI water $1: 3 \mathrm{v} / \mathrm{v}, \rho \approx 1.08 \mathrm{~g} / \mathrm{cm}^{3}$ ) with $2 \%$ w/v surfactant and $\sim 4 \mathrm{~mL}$ of DI water with $2 \%$ w/v surfactant. This creates a step gradient. The linear gradient is then produced by diffusion: the cell is capped and tilted horizontally for $2 \mathrm{~h}$ and then vertically for a further 2 h. ${ }^{39,130}$ This speeds selfdiffusion, stabilizing the gradient before ultracentrifugation. Separation is carried out via ultracentrifugation (Sorvall WX ultra100) in a TH641 swinging rotor at $40 \mathrm{kRPM}$ for $12 \mathrm{~h}$ at $15^{\circ} \mathrm{C}$, the same temperature as for ultrasonication. The average and maximum accelerations are $\sim 198000$ and $275000 \mathrm{~g}$. Fractionation is carried out as for section 2.3.2.

2.3.4. Metallic versus Semiconducting Separation. The $\mathrm{m} / \mathrm{s}$ separation is achieved in a cosurfactants mixture, based on the principle that surfactants with different chemical structure adsorb in a different way on $\mathrm{m} / \mathrm{s}$-SWNTs sidewalls, due to their different polarizability, ${ }^{138}$ resulting in different buoyant densities.

We consider LA SWNTs and a cosurfactant mixture of SC and SDS. For s-SWNTs separation, $2 \mathrm{mg}$ of SWNTs are dispersed in $10 \mathrm{~mL}$ of DI water with $2 \% \mathrm{w} / \mathrm{v}$ SC. Ultrasonication and preultracentrifugation are carried out as for section 2.3.3. After preultracentrifugation, the upper $60 \%$ dispersion is decanted and then diluted into $2 \% \mathrm{w} / \mathrm{v}$ cosurfactant solution. This, after dilution with the preultracentrifuged dispersion, contains $1.6 \% \mathrm{w} / \mathrm{v}$ SC and $0.4 \% \mathrm{w} / \mathrm{v}$ SDS. The density gradient is formed in a Seton ultraclear open-top, $13 \times 51 \mathrm{~mm}, 5.0 \mathrm{~mL}$ cell with $2 \% \mathrm{w} / \mathrm{v}$ surfactant $(1.6 \% \mathrm{w} / \mathrm{v} \mathrm{SC}$ and $0.4 \% \mathrm{w} / \mathrm{v} \mathrm{SDS})$. A total of $0.5 \mathrm{~mL}$ of $100 \%$ Optiprep with $2 \% \mathrm{w} / \mathrm{v}$ surfactant is used as stopping layer. Then $1.2 \mathrm{~mL}$ of $50 \%$ Optiprep $(\rho \approx$ $1.16 \mathrm{~g} / \mathrm{cm}^{3}$ ) with $2 \% \mathrm{w} / \mathrm{v}$ surfactant is placed over the stopping layer. On top of this, $0.8 \mathrm{~mL}$ SWNTs dispersion (density adjusted to $1.12 \mathrm{~g} / \mathrm{cm}^{3}$ by addition of $100 \%$ Optiprep with $2 \%$ $\mathrm{w} / \mathrm{v}$ surfactant) is inserted. The cell is then filled with two more layers of Optiprep with different concentrations: $0.75 \mathrm{~mL}$ of $25 \%$ Optiprep $\left(\rho \approx 1.08 \mathrm{~g} / \mathrm{cm}^{3}\right)$ with $2 \%$ w/v of surfactant and $\sim 1 \mathrm{~mL}$ of DI water with $2 \% \mathrm{w} / \mathrm{v}$ surfactant. The linear gradient is produced by diffusion, as described in section 2.3.3.

For $\mathrm{m}$-separation, the dispersion processes and the formation of the density gradient follow the same protocol as for s-separation. The only difference is the change of the relative concentration of the cosurfactant mixture. After ultracentrifugation, the SC dispersion is diluted with a solution containing $0.4 \% \mathrm{w} / \mathrm{v}$ SC and $1.6 \% \mathrm{w} / \mathrm{v}$ SDS. This same concentration is used for the DGM formation, with a procedure identical to that used for s-separation. m-separation is carried out with a MLS 50 swinging bucket rotor in a Beckman-coulter ultracentrifuge (Optima MAX 80) at $48 \mathrm{kRPM}$ for $12 \mathrm{~h}$ at $15^{\circ} \mathrm{C}$. The resulting average and maximum accelerations are $\sim 185000 \mathrm{~g}$ and $\sim 255000 \mathrm{~g}$. The fractionation process is carried out as for section 2.3.2.

2.3.5. Chirality Enrichment. Chirality enrichment of tubes with the same diameter is done by first separating s- from $\mathrm{m}$-tubes and then repeating diameter separation on the fractions of interest. This allows, for example, to selectively enrich $(6,5)$ with respect to $(7,4)$ and $(6,6)$. This is a two step procedure, involving a first DGU process in a cosurfactant mixture, for $\mathrm{m} / \mathrm{s}$ separation, followed by a second step, where SC is used to separate tubes with different diameter.

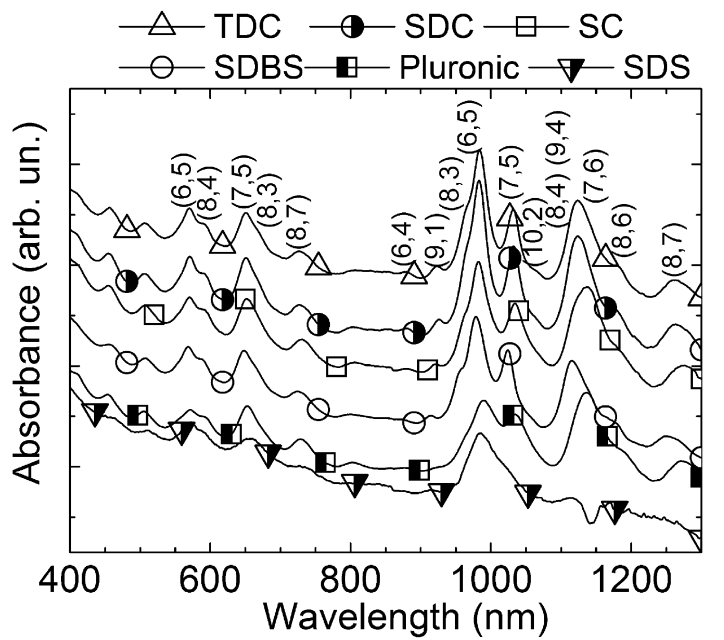

Figure 5. Absorption of CoMoCAT SWNTs dispersed in water using different surfactants after ultrasonication and ultracentrifugation $(\sim 173000 \mathrm{~g})$. The spectra are vertically shifted for clarity. The chiralities are assigned following ref 103.

For the first step $2 \mathrm{mg}$ of CoMoCAT tubes are dispersed in $10 \mathrm{~mL}$ of DI water with $2 \% \mathrm{w} / \mathrm{v}$ TDC. Dispersion and individualization proceed as for section 2.2. The dispersion is then diluted into $2 \% \mathrm{w} / \mathrm{v}$ cosurfactant solution (TDC-SDS) to achieve $1.6 \% \mathrm{w} / \mathrm{v}$ TDC and $0.4 \% \mathrm{w} / \mathrm{v}$ SDS. Then, a linear density gradient is prepared in 6 different cells as for section 2.3.3. The DGU and fractionation are then carried out as for sections 2.3.3 and 2.3.2.

The resulting s-SWNTs enriched fraction is inserted into a dialysis membrane (Spectra/Por Biotech Cellulose Ester (CE), MWCO: 3500) in order to remove the surfactants from the SWNT sidewalls. Osmotic phenomena govern dialysis. ${ }^{139,140}$ The membrane is immersed in a beaker containing $\sim 500 \mathrm{~mL}$ of DI water. The dispersion is stirred for 2 days, changing the DI water every $6 \mathrm{~h}$ to maintain the highest difference in concentration between the high surfactant concentration (inside the membrane) and low surfactant concentration (the beaker). During the process, water molecules pass through the membrane from the beaker, while the surfactant molecules go through the membrane in the opposite direction. At the same time, nanotubes, on average much longer than the membrane pore size, are retained. At the end of this process, tubes aggregate due to lack of surfactant molecules. In the second step, $2 \% \mathrm{w} / \mathrm{v}$ SC is added to the dialyzed dispersion and a new cycle is carried out as for section 2.2.

Linear density gradient preparation and DGU are as for section 2.3.3. The procedure can be iteratively used (multiple steps DGU) to improve single chirality enrichment.

2.4. Optical Characterization. 2.4.1. Optical Absorption Spectroscopy. Optical absorption spectra (OAS) reveal various properties such as transition energies, ${ }^{26,27,57,141,142}$ bundling, ${ }^{26,27,141,142}$ and concentration. ${ }^{63,68,70}$ For instance, sharp features in the absorption spectrum of CoMoCAT tubes (see Figure 5) are related to the first $\left(e h_{11} \approx 850-1250 \mathrm{~nm}\right)$ and second $\left(e h_{22} \approx\right.$ $500-850 \mathrm{~nm}$ ) excitonic transitions of s-SWNTs ${ }^{57,67,70}$ and first $\left(e h_{11} \approx 400-600 \mathrm{~nm}\right)$ excitonic transitions of m-SWNTs. ${ }^{57}$ Reference 143 reported a correlation of broadening and redshift of excitonic transition in bundles. This was assigned to the reduced strength of the effective Coulomb interactions due to intertube screening ${ }^{144,145}$ and can indicate bundle formation. ${ }^{26,143-146}$ The excitonic transitions are also strongly modulated by the dielectric environment. ${ }^{58,60,63,64,68,70,143,147-150}$ The latter can change as a result of noncovalent adsorption of 
solvents, ${ }^{63,68,70}$ gases ${ }^{147}$ or dispersants. ${ }^{58,60,64,71,143,150}$ For instance, an increase in dielectric screening modifies the electron-electron interactions, reducing the gap as reported in ref 149. Therefore, the ideal way to confirm the formation of SWNT bundles using OAS would be to compare a SWNTs dispersion with another containing only isolated SWNTs. However, it is not always possible to prepare such a reference sample and, consequently, determine the degree of individualization. Using the same solvent-surfactant combination ensures the same dielectric environment. Since here we employ different surfactants, relative shifts in absorption cannot be used as a signature of SWNT individualization.

The determination of the relative SWNT concentration of sorted sample is carried out taking the derivative of the absorption, as done by ref 72 , to more precisely determine the peak absorption. For quantitative analysis, if we assume that the absorption spectrum obeys the Beer-Lambert law, $A_{\lambda}=$ $\alpha_{\lambda} l c$, where $A_{\lambda}$ is the absorbance at wavelength $\lambda[\mathrm{nm}], \alpha_{\lambda}[\mathrm{L}$ $\left.\mathrm{mol}^{-1} \mathrm{~cm}^{-1}\right]$ is the absorption coefficient at the same wavelength, $l[\mathrm{~cm}]$ is the length of optical path, and $c\left[\mathrm{~mol} \mathrm{~L}^{-1}\right]$ is the concentration of material, there will be a similar relationship between concentration and amplitude for the first-order derivative: $\mathrm{d} A_{\lambda} / \mathrm{d} \lambda=\left(\mathrm{d} \alpha_{\lambda} / \mathrm{d} \lambda\right) l c$. Thus, the amplitude of the first-order derivative is proportional to the sample concentration. ${ }^{151}$

The advantages of derivative spectroscopy are (1) better spectral resolution; for example, $e h_{i i}$ optical transitions that are barely noticeable shoulders or even not resolved in the original spectrum are more clearly seen; (2) more precise determination of peak maxima; (3) elimination of baseline shift, because the first derivative of a constant is zero. Within this approach, we estimated the uncertainty on $(n, m)$ abundances as the standard deviation of 10 measurements on different samples for each sorting procedure.

We use a Perkin-Elmer Lambda 950 spectrophotometer. Measurements are carried out in the range 400-1300 nm, limited by the strong absorption features of Iodixanol and water. However, this is enough to cover the first and second excitonic transitions of s-SWNTs ${ }^{57,67,70}$ and first of m-SWNTs, for CoMoCAT. For LA, this covers the second and third transitions of s-SWNTs ${ }^{67,70}$ and first of m-SWNTs. Absorption from solvent and surfactants is subtracted, by measuring solutions with only solvent and surfactant.

The assignment of the optical transitions is based on the empirical Kataura plot of ref 103. This gives values of optical transition frequencies versus chirality for SWNT in aqueous surfactant dispersions, and is more appropriate than Kataura plots theoretically derived from tight binding or other models. We use this also for chirality assignment in photoluminescence excitation (PLE) and Raman spectroscopy.

2.4.2. Photoluminescence Excitation Spectroscopy. PLE is one of the most common techniques to monitor SWNT dispersions. ${ }^{26,63,68,152,153}$ The $\left(e h_{i i}, e h_{11}\right)$ resonances $(i=1,2, \ldots$, etc.) from different SWNTs appear as sharp features $\left(\lambda_{\mathrm{ex}}, \lambda_{\mathrm{em}}\right)$, where $\lambda_{\mathrm{ex}}$ and $\lambda_{\mathrm{em}}$ are the excitation and emission wavelengths, respectively. Other peaks can be observed, either due to excitonic-phonon sidebands, ${ }^{154-156}$ EET,,${ }^{26,27,157}$ or bright phonon sidebands (BS) of dark K-momentum excitons. ${ }^{158}$ In particular, EET occurs in bundles when the excitation of large bandgap donor SWNTs (d-SWNT) $e h_{i i}$ induces emission from $e h_{11}$ of a smaller bandgap acceptor (a-SWNT) ${ }^{26,27}$ EET can thus identify the presence of small bundles. ${ }^{26,27}$ EET is also largely independent of perturbations induced by surrounding dielectric environments, since d- and a-SWNTs must be in the same bundle for
EET to happen. ${ }^{26,27,159}$ Indeed, the EET range is limited to a few nanometers and governed by Förster resonance. ${ }^{26,159}$

In general, the PL intensity is proportional to the concentration of a species, its absorption cross-section at the excitation wavelength, ${ }^{160}$ and its fluorescence quantum yield. Thus, it does not directly reveal the relative abundance of SWNTs. ${ }^{160}$ However, the relative PL intensity of different chiralities can be used to compare the effectiveness of their individualization by different surfactants.

PLE maps of the dispersions are recorded using a Horiba Jobin-Yvon excitation-emission spectrofluorometer (Fluorolog 3) with $10 \mathrm{~nm}$ slit for the double grating excitation monochromator and $14 \mathrm{~nm}$ for the single grating emission one. The scan step is $5 \mathrm{~nm}$ for excitation, with a range from 440 to $850 \mathrm{~nm}$ and from 600 to $980 \mathrm{~nm}$ for CoMoCAT and LA samples, respectively. These cover the $e h_{22}$ of these tubes. ${ }^{103}$ The emission is collected by a liquid-nitrogen-cooled InGaAs detector using a right angle scattering, in the 900-1200 $\mathrm{nm}$ and 900-1600 $\mathrm{nm}$ ranges for CoMoCAT and LA, respectively. Note that $\sim 1600 \mathrm{~nm}$ is our upper detection limit. The raw PL data are corrected by the excitation profile. All of the dispersions are diluted with their respective surfactant or cosurfactant mixture solutions to an absorbance of about 0.1 in the entire absorption spectrum, in order to avoid reabsorption effects.

2.4.3. Raman Spectroscopy. Raman spectroscopy can be used to probe SWNTs within dispersions. In the low frequency region, the radial breathing modes (RBMs) are observed. ${ }^{161}$ Their position $\operatorname{Pos}(\mathrm{RBM})$, is inversely related to SWNT diameter, $d,{ }^{162-164}$ as given by $\operatorname{Pos}(\mathrm{RBM})=\left(C_{1} / d\right)+C_{2}$. Combining $\operatorname{Pos}(\mathrm{RBM})$ with excitation wavelength and the "Kataura plot"103,165 it is, in principle, possible to derive the SWNT chirality. ${ }^{166,167}$ References 162-164 and 168 report tables where, for each $(n, m)$, the corresponding $\operatorname{Pos}(\mathrm{RBM})$ and transition energies are assigned. Matching the diameter given by $\operatorname{Pos}(\mathrm{RBM})$ with excitation wavelength in the Kataura plot also gives information on the s- or m-character. A variety of $C_{1}$ and $C_{2}$ were proposed for this relation. ${ }^{161-163,167,168}$ Here we use $C_{1}=214.4 \mathrm{~cm}^{-1} \mathrm{~nm}$ and $C_{2}=18.7 \mathrm{~cm}^{-1}$, from ref 162 . These were derived by plotting the resonance energy as a function of inverse RBM frequency without any additional assumptions. However, we also validated our results by using the parameters proposed in refs $163,168,169$. Reference 163 exploited a combination of electron diffraction and Raman scattering on the same SWNTs to directly measure the relation between RBM frequency and tube diameter. In ref 168 the third and fourth optical transitions (over broad diameter $(0.7-2.3 \mathrm{~nm})$ and energy $(1.26-2.71 \mathrm{eV})$ ranges) for SWNTs deposited on quartz were investigated. In ref 162 a single parameter $(\mathrm{Ce})$ was used to account for the diameter-dependent environmental effects. ${ }^{169}$ For all excitation energies and samples analyzed, we found a maximum discrepancy of $0.03 \mathrm{~nm}$ on the tube diameter.

Raman spectroscopy also probes possible damage via the $D$ peak. ${ }^{170-172}$

The typical Raman spectrum in the $1500-1600 \mathrm{~cm}^{-1}$ region consists of the $\mathrm{G}^{+}$and $\mathrm{G}^{-}$bands. In s-SWNTs, they originate from the longitudinal (LO) and tangential (TO) modes, respectively, derived from the splitting of the $E_{2 g}$ phonon of graphene. ${ }^{173-176}$ The positions of the $\mathrm{G}^{+}$and $\mathrm{G}^{-}$peaks, $\operatorname{Pos}\left(\mathrm{G}^{+}\right)$ and $\operatorname{Pos}\left(\mathrm{G}^{-}\right)$, are diameter dependent and the separation between them increases with decreasing diameter. ${ }^{174,177}$ In m-SWNTs, the assignment of the $\mathrm{G}^{+}$and $\mathrm{G}^{-}$bands is the opposite, the Full Width at Half Maximum (FWHM) of the $\mathrm{G}^{-}$peak, $\operatorname{FWHM}\left(\mathrm{G}^{-}\right)$, is larger and $\operatorname{Pos}\left(\mathrm{G}^{-}\right)$down-shifted with respect to the semiconducting counterpart. ${ }^{173,174}$ Thus, a wide, low 
frequency $\mathrm{G}^{-}$is a fingerprint of $\mathrm{m}-\mathrm{SWNT}$. On the other hand, the absence of such feature does not necessarily imply that only $\mathrm{s}-\mathrm{SWNT}$ are present, but could just signify that m-SWNTs are off-resonance.

Doping could also modify positions and FWHMs. ${ }^{178-180}$ In m-SWNTs, a $\operatorname{Pos}\left(\mathrm{G}^{-}\right)$blueshift, accompanied by a FWHM $\left(\mathrm{G}^{-}\right)$decrease, is observed with electron or hole doping. ${ }^{178,179}$ In s-SWNTs, doping upshifts $\operatorname{Pos}\left(\mathrm{G}^{+}\right)$but does not affect $\mathrm{FWHM}\left(\mathrm{G}^{+}\right) \cdot{ }^{178,179}$

Thus, a large number of excitation wavelengths are necessary for a complete characterization of SWNTs. ${ }^{164,168}$ Nevertheless, useful information can be derived even with few excitations, especially for process monitoring, when Raman compares the "raw" material with the end-product. In particular, we note that ref 181 reported that tubes up to $100 \mathrm{meV}$ off resonance from the excitation wavelength can be detected. Thus, for example, for $1.92 \mathrm{eV}$ excitation we are in resonance with $(7,5)$ and $(7,6)$, with diameter 0.82 and $0.86 \mathrm{~nm} .{ }^{181}$ For the same excitation wavelength it is also possible to see tubes $\sim 100 \mathrm{meV}$ offresonance such as $(11,1)$ and $(9,9)$, with diameter 0.9 and 1.22 nm. ${ }^{181}$ Thus, taking the relationship between $\operatorname{Pos}\left(\mathrm{G}^{+}\right), \operatorname{Pos}\left(\mathrm{G}^{-}\right)$ and diameter of ref 174 , the $(7,5)-(11,1)$ tubes would give a spread of $\operatorname{Pos}\left(\mathrm{G}^{+}\right)$and $\operatorname{Pos}\left(\mathrm{G}^{-}\right)$of $\sim 4$ and $7 \mathrm{~cm}^{-1}$, respectively. In the case of $(7,5)-(9,9)$ tubes, the spread is $\sim 10$ and $\sim 15$ $\mathrm{cm}^{-1}$ for $\operatorname{Pos}\left(\mathrm{G}^{+}\right)$and $\operatorname{Pos}\left(\mathrm{G}^{-}\right)$. It is important to note also that tubes in resonance with the same laser energy can have a different diameter. For example, at $2.43 \mathrm{eV},(8,2),(8,5)$, and $(7,7)$ are in resonance. ${ }^{181}$ The diameter of $(8,2)$ is $0.72 \mathrm{~nm}$, whereas those of $(8,5)$ and $(7,7)$ are 0.89 and $0.95 \mathrm{~nm} .{ }^{181}$ Due to the diameter dependence of $\operatorname{Pos}\left(\mathrm{G}^{+}\right)$and $\operatorname{Pos}\left(\mathrm{G}^{-}\right),{ }^{174,177}$ we expect the removal of tubes with large difference in diameter to reduce the FWHM of both $\mathrm{G}^{+}$and $\mathrm{G}^{-} .{ }^{174,177}$

We measured the Raman spectra on both raw material and on sorted dispersions. The powders are deposited on an $\mathrm{Al}$ substrate, to avoid any Raman background. The dispersions are spin-cast on $\mathrm{Al}$ substrates and left to evaporate at room temperature overnight. Micro Raman spectra are acquired with a Renishaw 1000 at $488 \mathrm{~nm}(2.54 \mathrm{eV}), 514 \mathrm{~nm}(2.41 \mathrm{eV}), 633$ $\mathrm{nm}(1.96 \mathrm{eV})$, and $785 \mathrm{~nm}(1.58 \mathrm{eV})$, using a $100 \times$ objective and less than $1 \mathrm{~mW}$ on the sample. The RBM detection is limited by the cutoff of the notch and edge filters. These are at 130, 230, 110, and $120 \mathrm{~cm}^{-1}$ for 488, 514, 633, and $785 \mathrm{~nm}$. Thus, while at 488,633 , and $785 \mathrm{~nm}$ we detect tubes with diameter up to $1.9 \mathrm{~nm}$, at $514.5 \mathrm{~nm}$ we cannot detect tubes with diameter $>1.0 \mathrm{~nm}$. We use Lorentzians to fit RBM, $\mathrm{G}^{-}$and $\mathrm{G}^{+}$ peaks.

\section{Results and Discussion}

3.1. SWNTs Individualization. Figure 5 plots the absorption spectra of the six surfactant encapsulated SWNT dispersions. The $(6,5)$ has sharp $e h_{11}$ profiles in TDC, SDC, SC, and SDBS but broader in Pluronic F98 and SDS, with FWHM 75\% and $\sim 100 \%$ larger than SDC. Further, the $e h_{11}$ of $(10,2),(7,6),(9,4)$, and $(8,4)$ is not resolved in SDS. Hence, we conclude that those tubes are not well dispersed by this surfactant. Smaller diameter SWNTs (e.g., $(6,4)$ and $(9,1))$ show sharp features in bile salts, indicative of their effective individualization. In contrast, they have weak peaks in Pluronic F98.

Figure 6 plots the absorbance of the $(6,5) e h_{11}$. The $(6,5)$ have the highest population in as-grown CoMoCAT samples. ${ }^{181}$ Considering that the $(6,5) e h_{11}$ absorption is similar in all six dispersions, even though the peak position varies from 977 to $981 \mathrm{~nm}$ due to dielectric environment ${ }^{58}$ and/or bundling, ${ }^{12,57,144}$ we assume that the absolute absorbance of the $(6,5) e h_{11}$ will

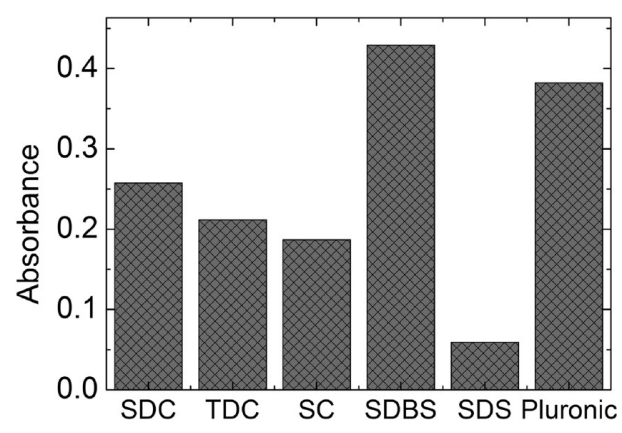

Figure 6. Absorbance of $(6,5)$ tubes dispersed in water using different surfactants, derived from the measurements in Figure 5.

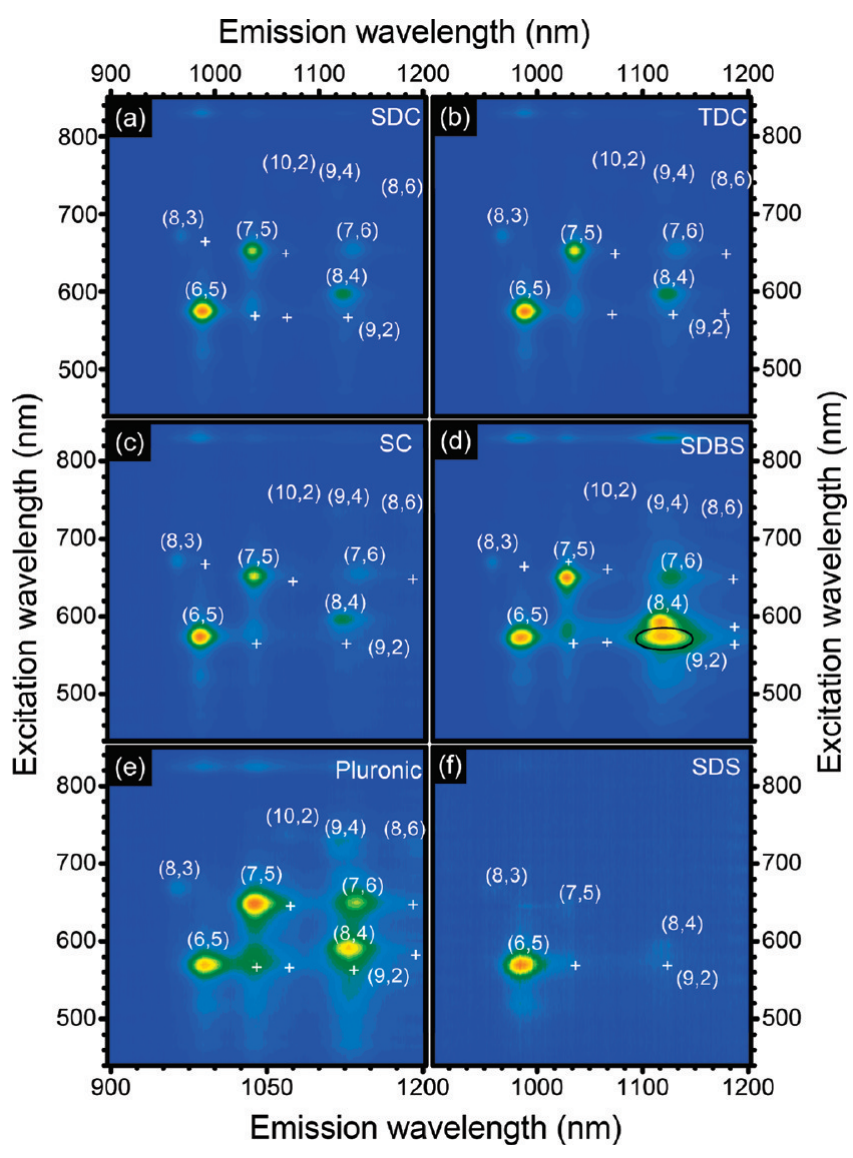

Figure 7. PLE maps from CoMoCAT SWNTs dispersed using the surfactants shown in Figure 2. (a) SDC, (b) TDC, (c) SC, (d) SDBS, (e) Pluronic F98, and (f) SDS. The chiralities are assigned following ref 103. Crosses represent EET features. ${ }^{26}$ The ellipse in (d) highlights EET from $(6,5)$ to $(8,4),(9,4)$, and $(7,6)$.

be proportional to their concentration. By comparing the $(6,5)$ $e h_{11}$ absorbance among all dispersions, we get a qualitative estimate of the relative amount of dispersed tubes. Figure 6 thus indicates that SDBS and Pluronic F98 disperse more tubes, since their $(6,5) e h_{11}$ absorbance is almost twice that of bile salts. We thus infer that they contain almost twice individual SWNTs compared to bile salts. Figure 6 also shows that the $(6,5) e h_{11}$ absorbance in SDS is $\sim 7$ times lower than SDBS, suggesting that SDS is not ideal for DGU, due to low concentration of SWNTs after the preultracentrifugation step.

Figure $7 \mathrm{a}-\mathrm{f}$ shows PLE maps of ultracentrifuged (122 000g) dispersions in the six surfactants. PL from BS satellites ${ }^{158}$ is not observed because these are very weak and can be partially overlapped by other, much stronger, features, such as excitonexciton resonances of tubes with different chiralities, phonon sidebands, or EET. ${ }^{26,27}$ Figure $7 \mathrm{a}-\mathrm{c}$ shows that bile salts 


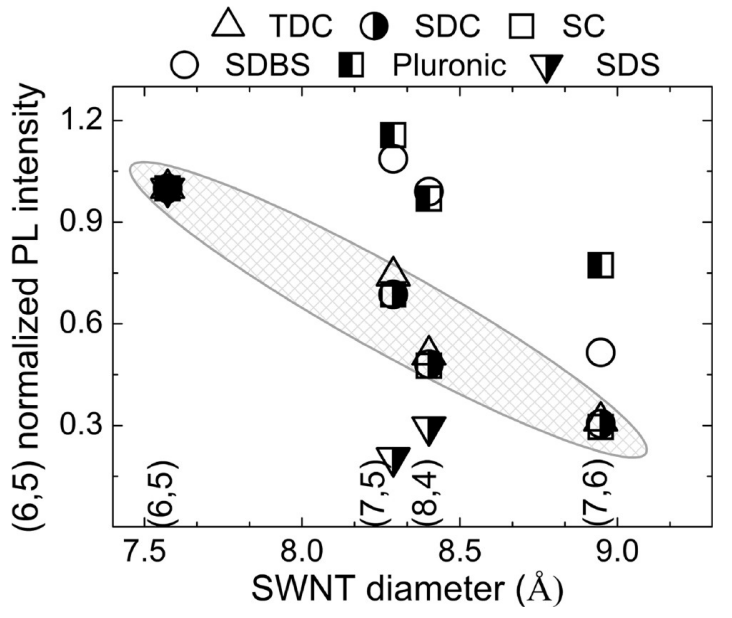

Figure 8. Selectivity of different surfactants in isolating different SWNT species. The PL intensity is normalized to the $(6,5)$ one. The hatched area includes the normalized PL intensities of bile salts.

dispersions have similar relative PL for different species. In contrast, only $(6,5)$ have detectable PL in SDS (Figure 7f). This is not a consequence of SDS selectivity toward $(6,5)$ but, as demonstrated by the above absorption analysis, is due to the low performance in individualization and debundling of SDS. Thus, the fact that mainly $(6,5)$ are detected in the corresponding PLE map (Figure 7f) is due to their higher concentration with respect to the other chiralities in the starting material.

Figure 8 plots the PL intensities of all chiralities normalized to $(6,5)$. It shows a PL decrease with diameter for bile salt, in contrast to SDBS and Pluronic F98. For example, the relative PL intensities of $(7,5),(8,4)$ and $(7,6)$ in bile salts are $\sim 70 \%$, $\sim 50 \%$, and $30 \%$ of $(6,5)$. However, in Pluronic F98, the relative PLs of the same tubes with respect to $(6,5)$ are $\sim 115 \%, \sim 95 \%$, $\sim 80 \%$, respectively. We achieve similar results also with SDBS (see Figure 8). Thus, Pluronic F98 and SDBS tend preferentially to disperse tubes with diameter larger than $0.75 \mathrm{~nm}$. The EET features in the PLE maps of Figure 7 are marked by solid crosses. The dispersion with SDBS shows the strongest EET $\left(e h_{22}^{\mathrm{D}}, e h_{11}^{\mathrm{A}}\right)$ from $(6,5)$ to $(7,6),(8,4)$, and $(9,4)$ at $\sim 1120 \mathrm{~nm}$, Figure $7 \mathrm{~d}$. This is so strong that it is comparable to the $\left(e h_{22}, e h_{11}\right)$ emission from $(8,4)$. On the other hand, the $\left(e h_{22}^{\mathrm{D}}, e h_{11}^{\mathrm{A}}\right)$ emissions for the same donor-acceptor pairs in bile salts are very weak, see Figure $6 a-c$. This shows the inefficiency of SDBS in individualizing SWNTs compared to bile salts. Note that the absorption spectra (Figure 5) provide little or no information on bundling, because both dispersions with SDBS and SC have a similar absorption profile and FWHM.

3.2. Diameter Separation. 3.2.1. Effect of Bundling and Surfactant Coverage. The inefficient individualization of SWNTs in SDBS discussed in section 3.1 is reflected in the ineffectiveness of the DGU separation. After DGU, the majority of SDBS encapsulated SWNTs (still bundled) reach their isopycnic point in proximity of the stopping layer $(60 \%$ iodixanol) as shown by the black band in Figure 9a. The lack of colored bands is a first indication of separation failure. Figure $9 \mathrm{~b}$ plots the absorption spectra of the fractionated aliquots from a SDBS encapsulated CoMoCAT dispersion. They have a similar profile from top to bottom, confirming no diameter separation. Contrary to previous reports on efficient dispersion of SWNTs in SDBS, ${ }^{58,59}$ we observe EET features (indicated by the arrow and crosses in Figure 9c) even from the topmost fraction. These are a signature of bundling, as discussed in section 2.4.2. ${ }^{26,27,159}$ Thus, even though SDBS can disperse twice

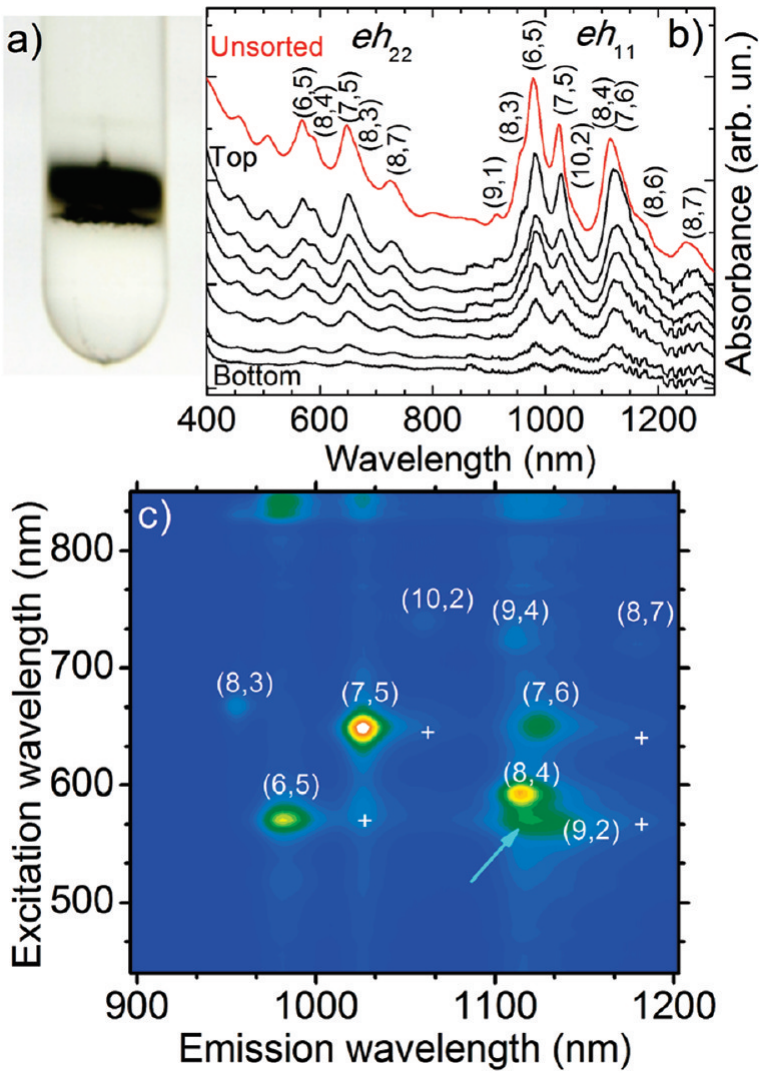

Figure 9. (a) Photograph and (b) absorption after DGU separation for SDBS encapsulated CoMoCAT SWNTs. The spectrum of the unsorted sample is also plotted (red line) for comparison. (c) PLE from the top fraction shows EET (arrow) from $(6,5)$ to $(8,4),(9,4)$, and $(7,6){ }^{26}$ The chiralities are assigned following ref 103 .

the amount of SWNTs compared to bile salts, it fails in their individualization, hindering further diameter separation.

Polymers are also commonly used as SWNT dispersants in water. ${ }^{12,58}$ In order to test their effectiveness in DGU, we carried out separation by diameter using Pluronic F98, which is known to disperse SWNTs in aqueous solutions. ${ }^{58} \mathrm{We}$ observe an improvement compared to SDBS, as shown by the two distinct and broad colored bands in Figure 10a. Figure 10b plots the absorption corresponding to different fractions. The bottom fraction (f15) has all the species expected from CoMoCAT SWNTs. ${ }^{105,106}$ Compared to the top fraction, the broadening $(\sim 16 \%)$ and the $5 \mathrm{~nm}$ red-shift of the $(6,5)$ peak suggest the presence of bundles, which cannot be separated by DGU. The absorption of the middle fraction (f9), in contrast, indicates a highly selective enrichment of $(8,4),(7,6),(9,4)$ with diameters in the $0.84-0.91 \mathrm{~nm}$ range. Fraction f5 shows $\sim 50$ enhancement of $(7,5)$ compared to the starting material. Figure 10c,d are PLE maps of f5 and f9. The f5 PLE has strongest emission from $(7,5)$. In comparison with the starting material, Figure $7 \mathrm{e}$, a strong reduction of emission from $(6,5)$ and $(8,3)$ is observed. On the contrary, the f9 map shows also larger diameter tubes, such as $(8,4),(9,2)$, and $(7,6)$.

The encapsulation mechanism of Pluronic F98 is different from that of anionic surfactants such as SDBS. ${ }^{110}$ In SDBS, charge repulsion is the dominating factor impeding SWNTs aggregation. ${ }^{58}$ In Pluronic F98 hydrophobic interactions ${ }^{116,182,183}$ link sidewalls with the polymer molecules. ${ }^{58}$ The adsorption of the Pluronic molecules could be due to the PPO chains binding to the tube sidewalls. The remainder of the chain, PEO, would project into the aqueous phase to form tails or loops. ${ }^{110,184,185}$ 

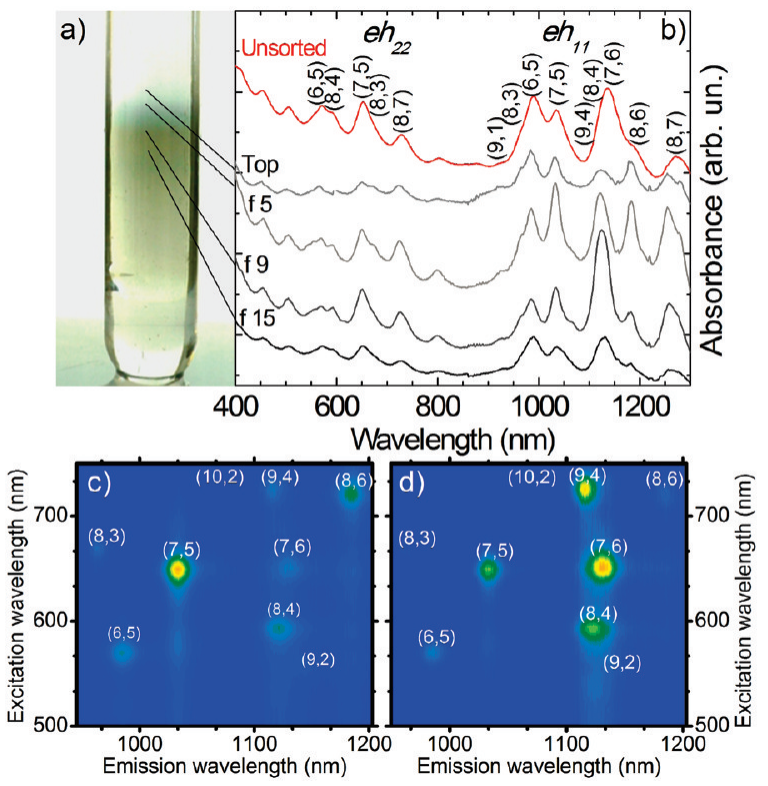

Figure 10. (a) Photograph and (b) optical absorption after DGU separation of the Pluronic F98-encapsulated CoMoCAT SWNTs dispersion. The spectrum of the unsorted sample is also plotted (red line) for comparison. (c and d) PLE maps of (c) fraction f5 and (d) fraction f9. The chiralities are assigned following ref 103.

The thickness of the adsorbed layer determines the strength of the steric repulsion between these groups, hindering aggregation with other SWNTs. ${ }^{186}$

If SWNTs are uniformly encapsulated by the surfactant, the steric stabilization (due to the formation of a film of adsorbed molecules at the surface of the SWNTs, hindering the approach of other SWNTs) is effective, producing individualized tubes. In this case, the buoyant density would depend only on diameter, thus allowing diameter separation.

Figure 11a is a cell after DGU of SWNTs encapsulated in dihydroxy TDC. The bottom fractions contain all chiralities, as seen from the absorption in Figure 11b (black line). On the other hand, absorption from the topmost fraction in Figure 11b (gray line) has a lower background and a strong band at $983 \mathrm{~nm}$, corresponding to the $(6,5) \mathrm{eh}_{11} \cdot{ }^{103}$ Figure $11 \mathrm{c}$ shows higher PL from $(6,5)$, compared to other tubes, while no PL is detected from tubes in the diameter range $0.9-1.0 \mathrm{~nm}$. The percentage of s-SWNTs in the diameter range $0.76-0.78$ is $\sim 57 \%$. The other features correspond to $(6,4),(7,5),(7,6),(8,4),(9,2),(8,6)$. However, we stress this is a qualitative estimation, because a fully quantitative $(n, m)$ evaluation in a given sample is still not possible, since this would require the knowledge of extinction coefficients and quantum yields for each $(n, m)$.

The effectiveness of the separation processes is related to the surfactant structure. Typical anionic and nonionic surfactants form ellipsoidal or spherical micelles with a hydrocarbon interior. ${ }^{111,114}$ In contrast, bile salts are steroids with a rigid cyclopentenophenanthrene nucleus ${ }^{115,116}$ and are shaped like a flattened ellipsoid with dissimilar sides. ${ }^{115,116}$ The $\beta$-face is hydrophobic, while the $\alpha$-face, with $-\mathrm{OH}$ groups, is hydrophilic. ${ }^{115,116}$ A short aliphatic chain protrudes from one end of the steroid nucleus and terminates in a strong hydrophilic group. ${ }^{187}$ Therefore, bile salts contain one hydrophobic side, one hydrophilic side and a short hydrophilic tail. ${ }^{115,116}$ Due to this structure, bile salts encapsulate SWNTs differently from linear chain surfactants, enabling better individualization and uniform sidewall coverage. For example, SDS was proposed to form cylindrical micelles ${ }^{57}$ and hemimicelles ${ }^{59}$ around tube sidewalls.

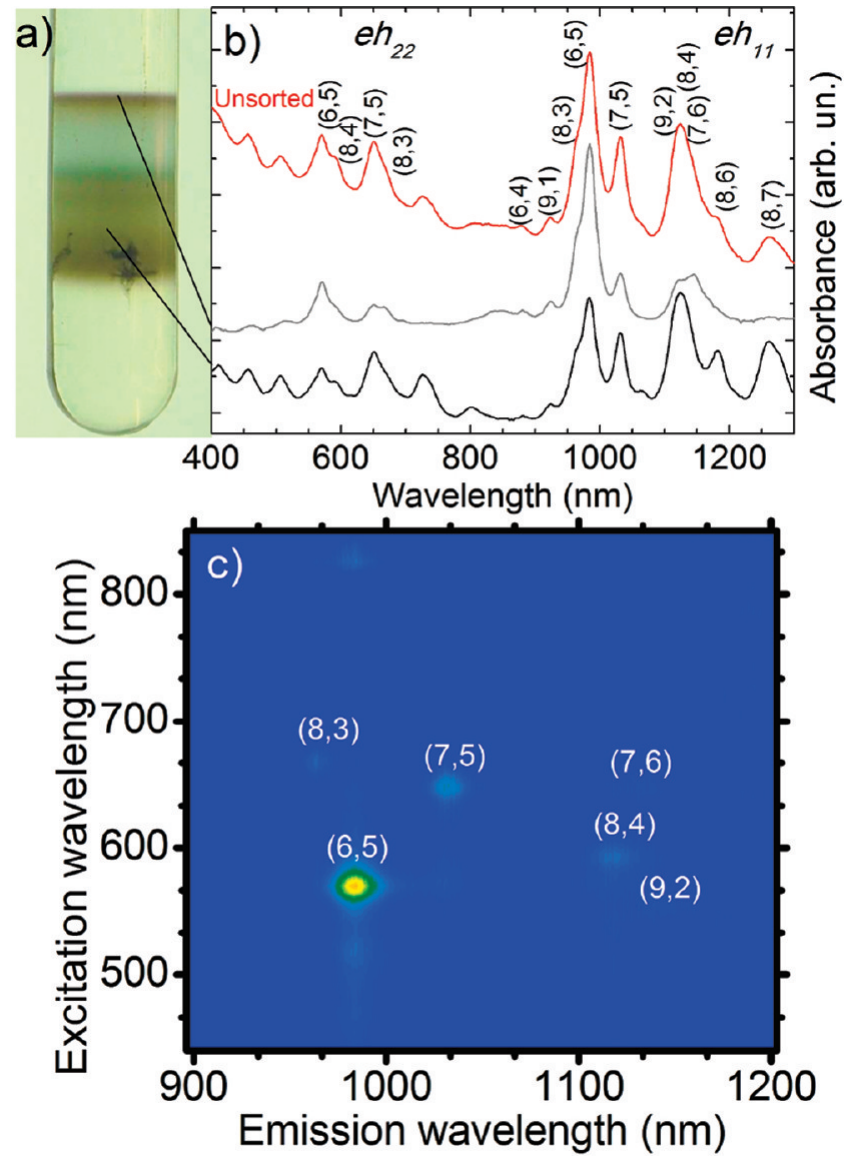

Figure 11. (a) Photograph and (b) optical absorption before (red line) and after DGU from TDC dihydroxy bile salts-encapsulated CoMoCAT SWNTs dispersion. (c) PLE map of the top fraction. The chiralities are assigned following ref 103.

However, ref 188 argued, through small-angle neutron scattering experiments, that SDS molecules do not show a preferential arrangement of head and tail on tube sidewalls. In the case of bile salt, the hydrophobic $\alpha$ side would get in close contact with the sidewalls, while the hydrophilic $\beta$ side would interface with the aqueous environment.

We propose that the poor performance of linear chain surfactants in diameter separation, with respect to bile salts, is related, other than to inefficient debundling, to their surface coverage of SWNTs. Linear chain surfactants could form micelles around SWNTs with a random number of molecules, similar to their behavior with hydrophobic particles in aqueous solutions. ${ }^{116}$ Since DGU is sensitive to the buoyant density of the SWNT-surfactant assembly, a uniform surface coverage of the sidewalls is critical. This is why linear chain surfactants are less effective.

Figure 12a is a cell after the DGU first iteration using SDC. The OAS of one of the top fractions shows enrichment of small diameter tubes such as $(6,5)$ and $(9,1)$, with $d \approx 0.76 \mathrm{~nm}$, Figure $12 \mathrm{~b}$. It also reveals other tubes in the diameter range $0.68-0.89$ $\mathrm{nm}$. On the contrary, the bottom fractions are enriched with larger diameter tubes $(>1.0 \mathrm{~nm})$ and small bundles. The PLE map in Figure 12c shows strong PL emission from $(6,5)$, but PL from larger tubes such as $(8,3),(7,5),(8,4)$, and $(9,2)$ is also detected. Better diameter separation is achieved with the trihydroxy SC bile salt. Figure 12d shows a purple, narrow band. The absorption from one of the topmost fractions of this band (Figure 12e) has a strong signal from $(6,5)$ at $983 \mathrm{~nm}$ and negligible 

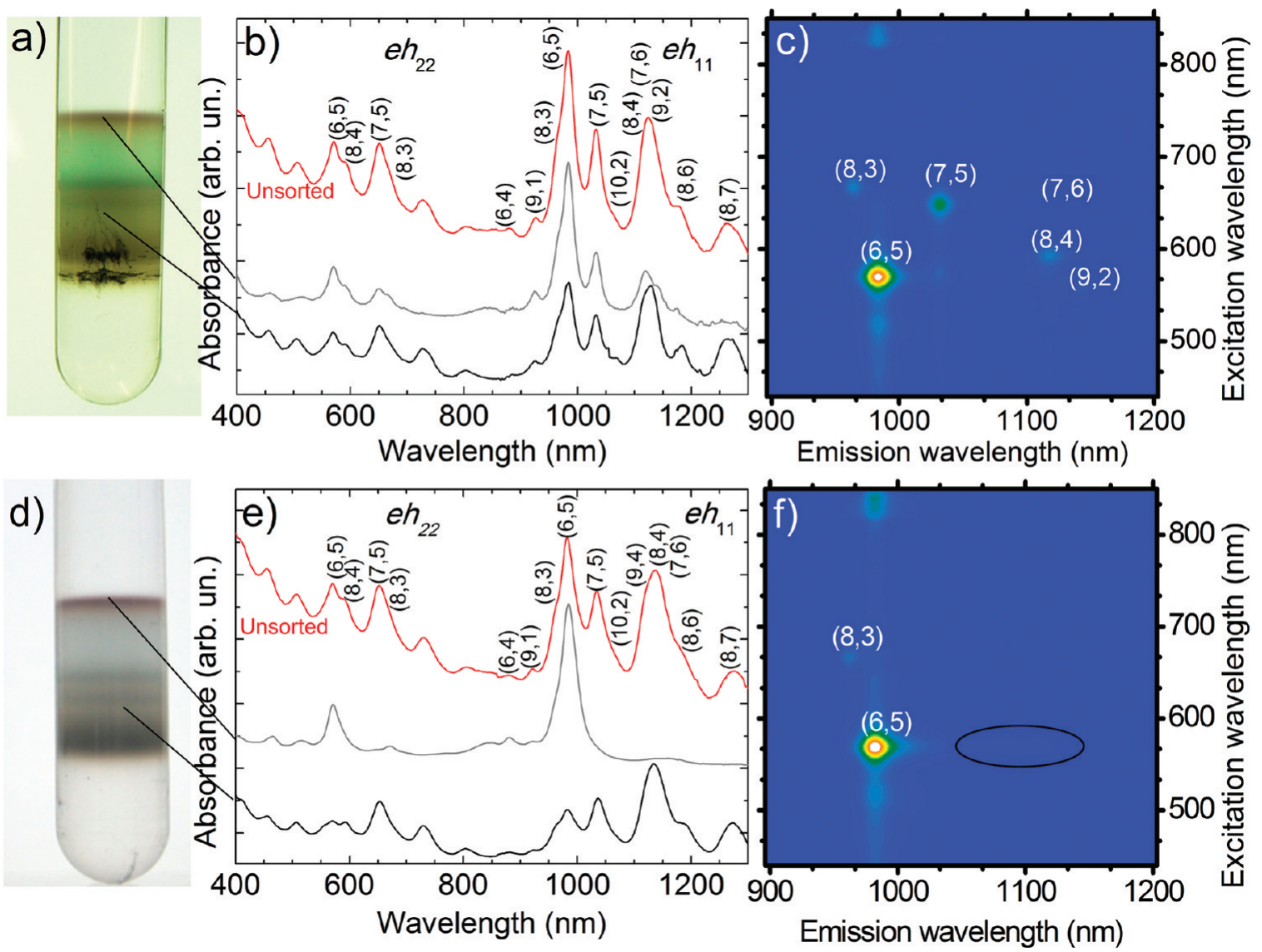

Figure 12. (a) Photograph and (b) absorption after DGU from a SDC-encapsulated CoMoCAT SWNT dispersion. The absorption from the unsorted material is plotted as well (red line). (c) PLE map of the topmost fraction. (d) Photograph of the ultracentrifuge cell after DGU using SC trihydroxy bile salt. (e) Absorption from the unsorted material (red line), topmost and one of the bottom fractions. (f) PLE of the topmost fraction. The ellipse indicates the $(6,5) e h_{11}-\mathrm{K}$ emission satellite. ${ }^{158}$ The chiralities are assigned following ref 103.

from larger diameters both for $e h_{22}$ and $e h_{11}$. The absorption from $(6,5)$ at $983 \mathrm{~nm}$ is $\sim 80 \%$ of the total in the $800-1300 \mathrm{~nm}$ range. The other features in this frequency range are those of $(6,4)$ at 883 $\mathrm{nm},{ }^{103}(9,1)$ at $921 \mathrm{~nm},{ }^{103}$ and $(8,3)$ at $963 \mathrm{~nm} .{ }^{103}$ Other peaks are seen in the $400-525 \mathrm{~nm}$ range, related to $\mathrm{m}$ - tubes such as $(6,6)$, $(8,2)$, and $(7,4)$. The spectrum corresponding to one of the bottom fractions in Figure 12e shows features associated with a large range of SWNT species. In particular, it indicates enrichment of tubes with diameter $\sim 0.8-1.0 \mathrm{~nm}$.

The narrow diameter distribution of SWNTs in the top fraction of the SC enriched sample is confirmed by PLE (Figure 12(f)). This has a strong $\left(e h_{22}, e h_{11}\right)$ resonant emission from $(6,5)$ and weaker from $(8,3)$. Other features, related to BS emission satellites of the $(6,5) e h_{11}$ at around $(570 \mathrm{~nm}, 1100$ $\mathrm{nm})$ are also seen. These were also reported in aqueous dispersions of DNA-wrapped CoMoCATs sorted by ionexchange chromatography. ${ }^{158}$ Despite the high PL emission from $(6,5)$, these weak $e h_{11}-\mathrm{K}$ emission satellites are not easily detectable in PLE of TDC and SDC encapsulated SWNTs, Figure 12c. This demonstrates the need of a highly enriched dispersion to avoid them being overshadowed by exciton-exciton resonances, ${ }^{189}$ phonon sidebands, ${ }^{155}$ and EET. ${ }^{26,27}$

Figures 13-15 compare the Raman spectra of the starting material (CoMoCAT powder) with those of sorted samples.

Figure 13a,b plots the spectra measured at $514.5 \mathrm{~nm}$ for the starting material and the top fractions of the samples sorted using SC, SDC, and SDBS. This wavelength is expected to be in resonance with the majority of $\mathrm{m}$-SWNTs in CoMoCAT samples. ${ }^{181}$ The spectrum of the starting material shows features related to m- and s-SWNTs, ${ }^{162}$ in particular RBMs of $(8,5),(9,3)$,
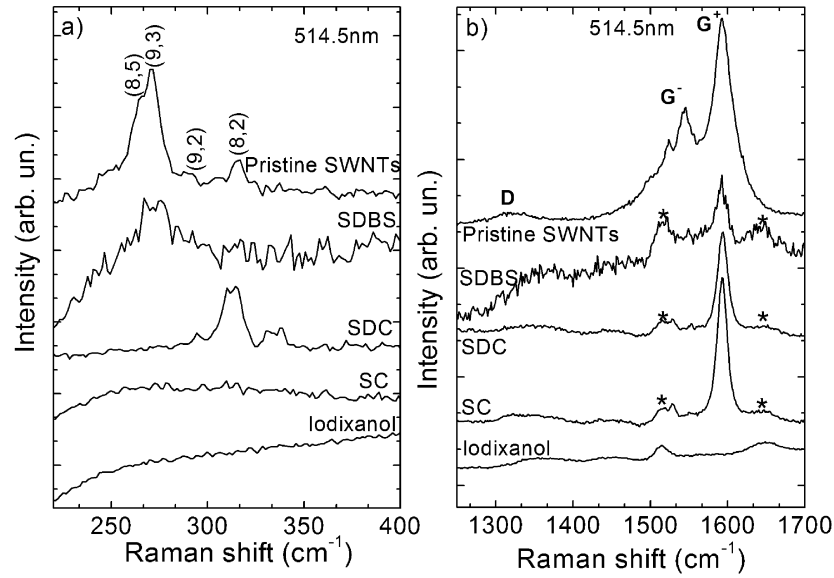

Figure 13. Raman spectra of CoMoCAT SWNTs measured at $514 \mathrm{~nm}$. (a) RBM and (b) G region. Spectra are offset for clarity. A reference iodixanol spectrum is also shown and its main features marked with *. The chiralities in (a) are assigned using $\mathrm{C}_{1}$ and $\mathrm{C}_{2}$ from ref 162, combining Pos(RBM) with excitation wavelength and the "Kataura plot". 103

$(9,2)$, and (8,2). After DGU, we still detect the RBMs of $(8,5)$ and $(9,3)$ in the SDBS sorted sample, while only the $(7,4)$ RBM is detected in SDC. On the contrary, no RBMs are detected in $\mathrm{SC}$ dispersion. We attribute the absence of the $(6,5) \mathrm{RBM}$ (the most abundant chirality both in absorption and PLE, see Figure $12, \mathrm{e}, \mathrm{f})$ to the fact that $514 \mathrm{~nm}$ is $\sim 0.24 \mathrm{eV}$ off-resonance with respect to the $(6,5) e h_{22}{ }^{103}$

In the $\mathrm{G}$ peak region, the starting material shows the typical $\mathrm{G}^{+}$and $\mathrm{G}^{-}$peaks expected from a mixture of s-and $\mathrm{m}-\mathrm{SWNTs}$ 

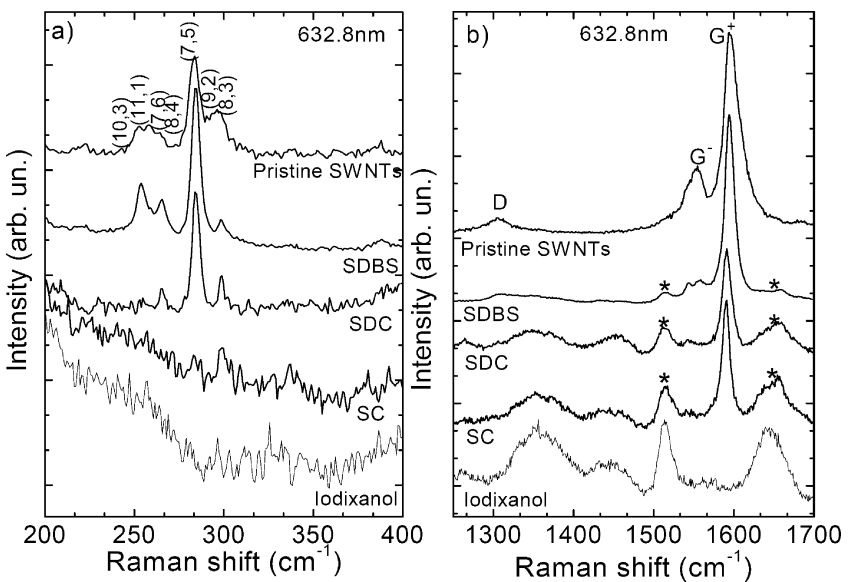

Figure 14. Raman spectra of CoMoCAT SWNTs measured at 633 $\mathrm{nm}$. (a) RBM and (b) G region. Spectra are offset for clarity. A reference iodixanol spectrum is also shown and its main features marked with*. The chiralities in (a) are assigned using $C_{1}$ and $C_{2}$ from ref 162 combined with the excitation wavelength and the "Kataura plot". ${ }^{103}$
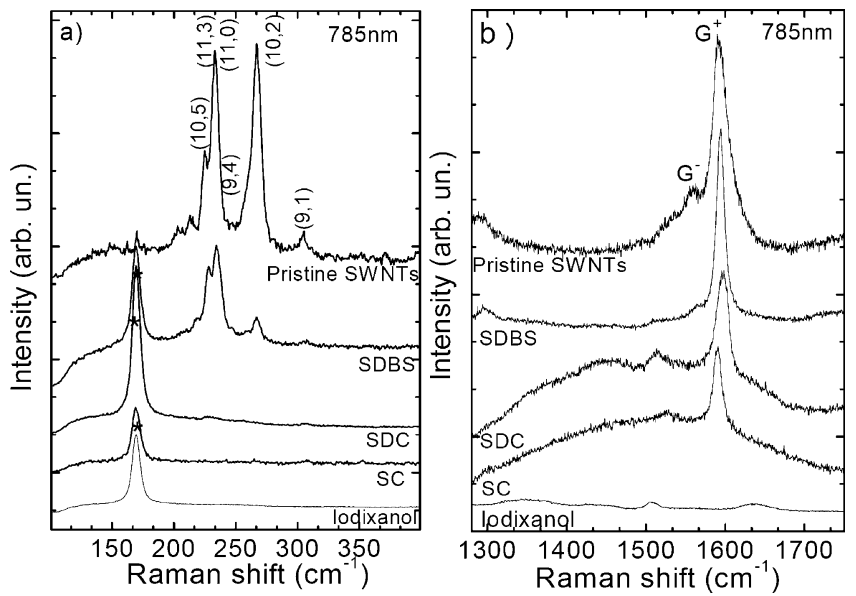

Figure 15. Raman spectra of CoMoCAT SWNTs measured at 785 $\mathrm{nm}$. (a) RBM and (b) G region. Spectra are offset for clarity. A reference iodixanol spectrum is also shown and its main features marked with $*$. The chiralities in (a) are assigned using $C_{1}$ and $C_{2}$ from ref 162, combined with excitation wavelength and the "Kataura plot". ${ }^{103}$

(see section 2.4.3). ${ }^{162,174,190} \mathrm{FWHM}\left(\mathrm{G}^{+}\right)$for the SC sample is narrower compared to the starting sample (14 instead of 33 $\mathrm{cm}^{-1}$ ). This indicates a narrower diameter distribution in $\mathrm{SC}$ : due to the diameter dependence of $\operatorname{Pos}\left(\mathrm{G}^{+}\right),{ }^{174,177}$ the removal of tubes with large difference in diameter will reduce $\operatorname{FWHM}\left(\mathrm{G}^{+}\right) .{ }^{174,177}$

Figure 14 plots the spectra measured at $633 \mathrm{~nm}$. In the pristine material, the RBMs of $(10,3),(11,1),(7,6),(8,4),(7,5),(9,2)$, $(8,3)$, and $(6,4)$ are seen, ${ }^{162,165}$ Figure $14 \mathrm{a}$. After DGU, in SDBS, $(10,3),(11,1),(7,6),(8,4),(7,5),(9,2)$, and $(8,3)$ are detected. ${ }^{162,165}$ These, except $(10,3)$ and $(11,1)$, are also seen in SDC. On the contrary, SC shows only RBMs at 299 and $337 \mathrm{~cm}^{-1}$, signature of $(8,3)$ and $(6,4) .{ }^{162,165}$ The starting material has $\mathrm{FWHM}\left(\mathrm{G}^{+}\right)$ $\sim 25 \mathrm{~cm}^{-1} ; \operatorname{Pos}\left(\mathrm{G}^{+}\right) \sim 1597 \mathrm{~cm}^{-1}$, Figure 14b. The SDBS sample has $\operatorname{FWHM}\left(\mathrm{G}^{+}\right) \sim 14 \mathrm{~cm}^{-1}$ and $\operatorname{Pos}\left(\mathrm{G}^{+}\right) \sim 1594 \mathrm{~cm}^{-1}$. The SDC and SC samples show a $\operatorname{FWHM}\left(\mathrm{G}^{+}\right) \sim 12 \mathrm{~cm}^{-1}$ and $\operatorname{Pos}\left(\mathrm{G}^{+}\right)$ $\sim 1590 \mathrm{~cm}^{-1}$. The SC sample has $\sim 7 \mathrm{~cm}^{-1} \operatorname{Pos}\left(\mathrm{G}^{+}\right)$downshift, and $14 \mathrm{~cm}^{-1} \mathrm{FWHM}\left(\mathrm{G}^{+}\right)$reduction compared to the raw material, implying a narrower diameter distribution.

Figure 15a plots the spectra measured at $785 \mathrm{~nm}$. In the pristine material, RBMs of $(10,5),(11,3),(11,0),(9,4),(10,2)$, and $(9,1)$ are detected. ${ }^{162,165}$ RBMs of larger tubes, such as $(10,5),(11,3)$, and $(11,0)$, are seen in SDBS, while only $(9,1)$ is detected in a)

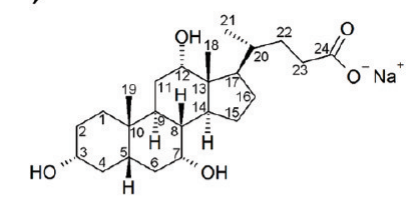

c)

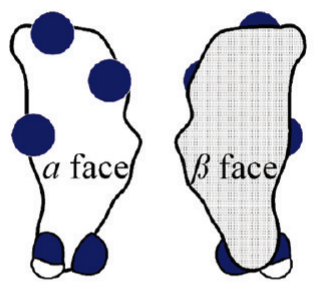

e)

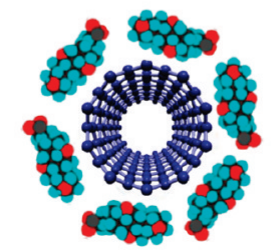

b) SDC

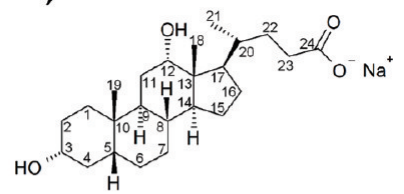

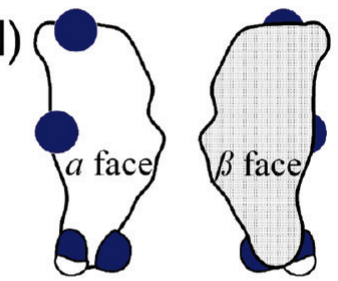

f)

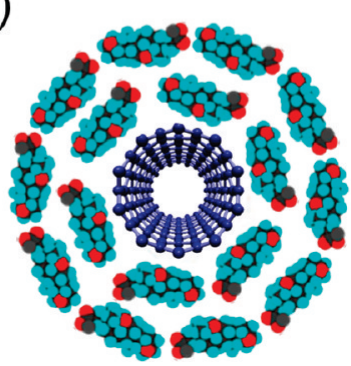

Figure 16. Molecular structure (adapted from ref 115) of (a) trihydroxy bile salt sodium cholate and (b) dihydroxy bile salt sodium deoxycholate. Model amphiphilic structure of (c) sodium cholate and (d) sodium deoxycholate. The side where the hydroxyl groups are located is hydrophilic $(\alpha)$, while the other is hydrophobic $(\beta)$. Schematic representation of micelle formation for (e) sodium cholate and (f) sodium deoxycholate.

SC. ${ }^{162,165}$ The narrower diameter distribution in SC is reflected in a narrower FWHM $\left(\mathrm{G}^{+}\right) \sim 13 \mathrm{~cm}^{-1}$, Figure $15 \mathrm{~b}$.

To summarize, Figures $13-15$ indicate that after DGU, the dispersion with SC has the narrower diameter distribution, with only $(6,4),(8,3)$, and $(9,1)$ signatures in the RBM region. ${ }^{162,165}$ The other chiralities are not detected due to off-resonance excitation (i.e., $(6,5)$ tubes) and the filters cut off. The Raman analysis confirms the ineffectiveness of SDBS in diameter separation, in agreement with absorption and PLE of Figure 9.

Considering all the tubes detected by absorption, PLE and Raman spectroscopy, we estimate the final diameter spread to be from 0.69 to $0.81 \mathrm{~nm}$, i.e., from $(6,4)$ to $(6,6)$. To the best of our knowledge, this is the narrowest thus far reported for singlestep DGU. Indeed, ref 37 reported a $0.75-1.03 \mathrm{~nm}$ range after the first iteration, while it was $0.69-0.89 \mathrm{~nm}$ in ref 39 .

3.2.2. Effect of Micelle Formation. Although, as discussed in section 3.1, SDC and TDC show better performance in terms of individualization, $\mathrm{SC}$ has the highest selectivity in terms of diameter separation. This disagrees with ref 37, where similar performances were reported for the three bile salts.

Since SDC and SC differ only in the number of - $\mathrm{OH}$ groups, see Figure 16a, the latter should be a key parameter for efficient encapsulation of SWNTs. Reference 116 reported that trihydroxy bile salts form much smaller micelles (hydrodynamic radii, $R_{\mathrm{h}}$ $=1.0-1.5 \mathrm{~nm})$ than the corresponding dihydroxy ones $\left(R_{\mathrm{h}}=\right.$ $1.5-6.0 \mathrm{~nm}$ ), with aggregation number (i.e., the number of molecules present in a micelle) $\sim 3$ and $\sim 20$, respectively. In addition, dihydroxy and trihydroxy bile salts form micelles of different shapes. ${ }^{191}$ While dihydroxy bile salts have elongated structures, the trihydroxy ones form highly hydrated globular micelles. ${ }^{116}$ The bile salt monomer has planar polarity with one 
surface hydrophobic and the opposite hydrophilic, due hydroxyl and ionized polar groups, ${ }^{116}$ Figure 16b. Bile salt micelles form in two stages: ${ }^{116}$ first the primary micelle appears and then the interaction between the hydrophilic surfaces of the salt molecules results in secondary micelles. ${ }^{192}$ This process is ruled by a succession of stepwise polymerization reactions: ${ }^{116,193}$

$$
\begin{gathered}
\mathrm{M}_{\mathrm{n} 0}+\mathrm{M}_{\mathrm{n} 0} \leftrightarrow(\mathrm{K}) \mathrm{D}_{2 \mathrm{n} 0} \\
\mathrm{D}_{2 \mathrm{n} 0}+\mathrm{M}_{\mathrm{n} 0} \leftrightarrow(\mathrm{K}) \mathrm{S}_{3 \mathrm{n} 0} \\
\mathrm{~S}_{(\mathrm{i}-1) \mathrm{n} 0}+\mathrm{M}_{\mathrm{n} 0} \leftrightarrow(\mathrm{K}) \mathrm{S}_{\mathrm{in} 0}
\end{gathered}
$$

Here, $\mathrm{M}_{\mathrm{n} 0}$ represents the monomer, $\mathrm{D}_{2 \mathrm{n} 0}$ the dimer, $\mathrm{S}_{3 \mathrm{n} 0}$ a primary micelle, and $S_{\text {ino }}$ a secondary micelle, while $\mathrm{K}$ is the polymerization constant defined by ${ }^{116,193}$

$$
K=\exp \left[-\left(\mu_{2 n 0}^{0}-2 \mu_{n 0}^{0}\right) / R T\right]
$$

where $\mu_{n 0}^{0}(\mathrm{kcal} / \mathrm{mol})$ is the standard chemical potential of the monomer, $\mu_{2 n 0}^{0}(\mathrm{kcal} / \mathrm{mol})$ that of the dimer, and $R(\mathrm{kcal} / \mathrm{K} \mathrm{mol})$ and $T(\mathrm{~K})$ are the molar constant and the temperature, respectively. ${ }^{116,193}$ Note that, although the equilibrium constant for micelle formation depends slightly on aggregation number, ${ }^{194,195}$ for bile salts this also depends on parameters such as temperature, species, and concentration. ${ }^{116,192}$ We thus assume the polymerization constant to be the same for each reaction involved in eq 1 and express it in terms of the dimerization of two primary micelles.

We suggest that bile salts and SWNTs form mixed micelles. The primary micelles are formed around the SWNT sidewalls, maintaining the monomer shape, exploiting the high hydrophobic nature of SWNTs. The $\beta$-side (the hydrophobic one) of the bile salts contacts the SWNT sidewalls. Therefore, the formation of the primary micelle, as monomer, on the SWNT sidewalls, does not depend on the number of $-\mathrm{OH}$ groups. The lack of the $-\mathrm{OH}$ group in position $\alpha 7$, Figure 16a,b, increases the hydrophobic area, initiating the secondary micelles. Experimentally, we measured an increase of the buoyant density of the top fraction (highly enriched in $(6,5))$ of the SC encapsulated SWNTs $\left(\sim 1.06 \mathrm{~g} / \mathrm{cm}^{3}\right)$ with respect to SDC and TDC $\left(\sim 1.045 \mathrm{~g} / \mathrm{cm}^{3}\right)$. We take this as evidence of a larger number of surfactant molecules around the SWNT sidewalls, which causes a reduction in the van der Waals forces between SWNT sidewalls. This screening, provided by the secondary micelles, may explain why SDC and TDC show better performance in SWNT individualization. On the contrary, the presence of the third $-\mathrm{OH}$ group reduces the formation of secondary micelles. ${ }^{116}$ Reference 116 attributed this to the total number of $-\mathrm{OH}$ groups and their position on the steroid backbone, which changes the hydrocarbon-water contact area. In fact, for $\mathrm{SC}$, the $-\mathrm{OH}$ group in position $\alpha 7$ (Figure 16a,b) on the hydrophilic part reduces the hydrocarbon-water contact area by $30-40 \%$, compared to dihydroxy species. ${ }^{116}$ The third $-\mathrm{OH}$ on the $\alpha$-side gives a smaller $\Delta \mu^{0}(\mathrm{kcal} / \mathrm{mol})$ change with respect to the dihydroxy species: ${ }^{116}$

$$
\Delta \mu^{0}=\Delta \mu_{\mathrm{EI}}+\Delta \mu_{\text {solvation }}+\Delta \mu_{\mathrm{H} \text { bond }}+\Delta \mu_{\mathrm{HI}}
$$

where $\Delta \mu_{\mathrm{EI}}$ is the free energy of electrostatic repulsion ${ }^{116}$ (a positive quantity), which must be overcome when two charged monomers and/or primary micelles are brought in contact. ${ }^{116} \Delta \mu_{\text {solvation }}$ is the energy difference associated with the solvation of micelles (a positive quantity). ${ }^{116}$ On the other hand, $\Delta \mu_{\mathrm{H} \text { bond }}$ and $\Delta \mu_{\mathrm{HI}}$ represent the free-energy change for intermicellar bonding between - $\mathrm{OH}$ groups on the micellar surfaces and hydrophobic interaction for the dimerization of the primary micelles. ${ }^{116}$ Both are negative. ${ }^{116}$ The formation of the secondary micelles is favored by the two negative quantities, $\Delta \mu_{\mathrm{H} \text { bond }}$ and $\Delta \mu_{\mathrm{Hr}}$. In particular, the driving force for the secondary micelle formation is the hydrophobic interaction between water molecules and the surface of the monomer or dimer. ${ }^{116}$ The $-\mathrm{OH}$ group in position $\alpha 7$ on the hydrophilic part of SC reduces the hydrocarbon-water contact area in comparison with SDC and TDC. ${ }^{116}$ Consequently, we believe that the reduced polymerization of $\mathrm{SC}^{116,187}$ hinders the formation of secondary micelles around the SWNT sidewalls. This determines uniform SC monolayer coverage, see Figure 16c, resulting in better DGU separation. On the contrary, the other two bile salts provide better individualization due to the large amount of secondary micelles (better screening), but with lower performance in separation. Indeed, a nonuniform coverage due to large micelles determines a change in buoyant density not related to the tubes themselves: the density variation among SWNTs of slightly different diameters could be compensated by the surfactant, resulting in poorer separation.

3.3. Metallic versus Semiconducting Separation. Section 3.2 showed that both individualization and surfactants aggregation are key to effective diameter separation. To separate mfrom s-SWNTs, the buoyant densities need to be made different according to the $\mathrm{m} / \mathrm{s}$ nature. This can be realized by tuning the relative concentration of the surfactants in a cosurfactant mixture. ${ }^{37,41,48}$ Thus, $\mathrm{m} / \mathrm{s}$ separation requires first the use of a primary surfactant to achieve individualization, then a secondary surfactant, mixed with the primary after preultracentrifugation, to induce a change in buoyant density between $\mathrm{m}$ - and s-SWNTs. The secondary surfactant must have a different molar volume with respect to the primary, to produce the highest possible change in buoyant density between $\mathrm{m}$ - and s-SWNTs.

We separate $\mathrm{m}$ - from s-SWNTs by exploiting a cosurfactant mixture of a bile salt (SC) and a linear chain surfactant (SDS). SC is used as primary surfactant due to its high performance in the diameter separation (see section 3.2), while SDS is used since it has a lower molar volume than SC. A second surfactant with smaller molar volume causes a change in the buoyant density of the tube-surfactant complex due to two possible reasons: (i) desorption of bile salt molecules from SWNT sidewalls, with subsequent adsorption of SDS, and (ii) adsorption of SDS onto a vacant area on the sidewalls, due to nonuniform bile salt coverage. In the first case, SC desorption from larger diameter tube sidewalls is favored because of the smaller activation energy required. In the second case, as m-SWNTs interact with the surfactants more strongly than s-SWNTs, due to their larger polarizability, ${ }^{196}$ the SDS adsorption onto vacant sites is favored, resulting in a buoyant density increase. The separation can be optimized by working on the cosurfactants ratio.

Since the excitonic transition energies of s- and m-SWNTs are grouped separately, ${ }^{103,197}$ OAS is ideal to characterize mand s-SWNT separated samples. ${ }^{37,42,65}$ Figure 17 shows an example of $\mathrm{m} / \mathrm{s}$ separation using LA SWNTs with a SC-SDS mixture (ratio 4:1). After DGU, two well-defined bands (orange on top of a dark cyan band) appear, Figure 17a, with a third band, black, at higher density.

Mixing the starting material after preultracentrifugation with a SC excess solution (SC:SDS-4:1), separates s-SWNTs, as seen in the absorption spectrum of Figure 17b. The $e h_{22}$ absorption $(750-1050 \mathrm{~nm}$ ) of s-SWNTs in the top fraction (orange band) 


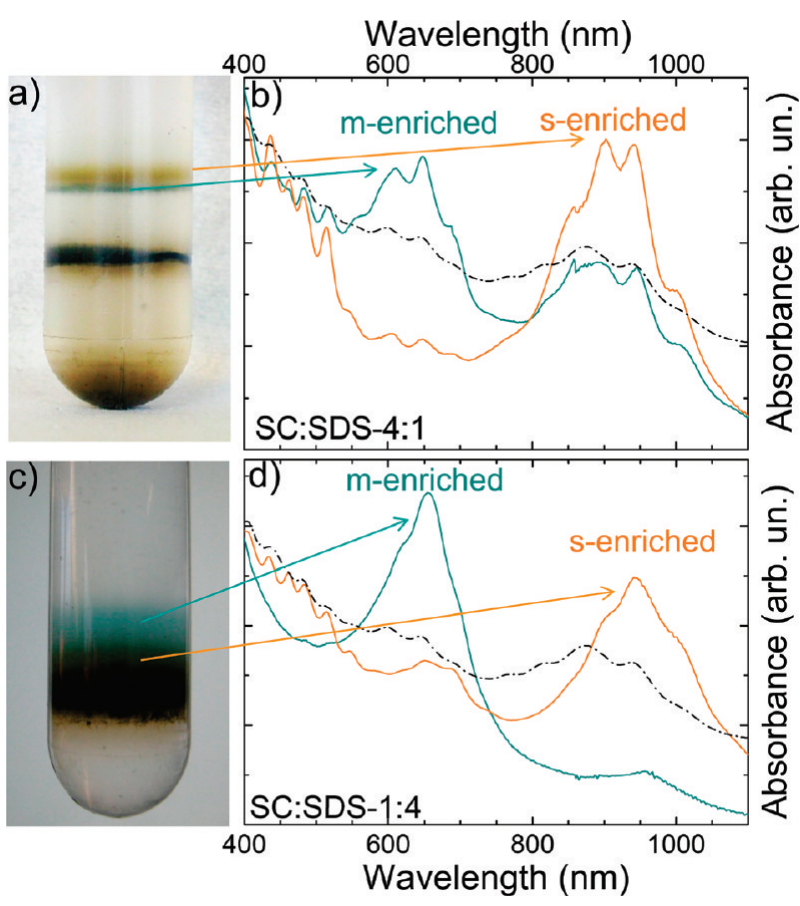

Figure 17. (a) Photograph of LA SWNTs sorted using a SC-SDS (ratio 4:1) mixture. (b) Absorption of fractions collected from the orange and dark cyan band of the SC-SDS:4-1 dispersion. (c) Photograph of the sorted SWNTs with a mixture SC-SDS (ratio 1:4). (d) Absorption of aliquots extracted from the orange and dark cyan band of the SC:SDS1:4 dispersion.

is enhanced, while the $e h_{11}$ of m-SWNTs $(550-750 \mathrm{~nm})$ is strongly reduced, with respect to the starting material. However, some absorption at 603, 646, and $689 \mathrm{~nm}$ is still present, signature of residual m-tubes. ${ }^{165}$ In Figure $17 \mathrm{~b}$ the absorption of the aliquots extracted from the dark cyan band is also plotted. Although an enhancement of m-SWNTs absorption is observed, some s-SWNTs remain, as confirmed by the bands in the $750-1050$ and 400-550 $\mathrm{nm}$ range, related to $e h_{22}$ and $e h_{33}$ of s-SWNTs, respectively. ${ }^{103}$

Better m-SWNTs separation is achieved tuning the cosurfactant mixture. Figure $17 \mathrm{c}$ shows the cell after DGU with a cosurfactant mixture SC-SDS (ratio 1:4). In this case, the m-SWNTs (large dark cyan band) stay at the top of the s-ones. We attribute the reduction in buoyant density of $\mathrm{m}$ - compared to s-SWNTs to the different surfactant arrangement on the sidewalls, due to different polarizability. The aggregation number of ionic surfactants was reported to increase with surfactant concentration. ${ }^{198,199}$ In SC:SDS-4:1, SDS is under the CMC, ${ }^{198}$ while for SC:SDS-1:4, it is over. ${ }^{198}$ Considering the higher polarizability of m-SWNTs, in the case of SC:SDS-1:4 the attachment of SDS could involve a bigger complex, depending on SDS micelle size. This consequently reduces the buoyant density of m-SWNTs with respect to s-SWNTs. Figure $17 \mathrm{~d}$ plots the absorption of the aliquot extracted from the dark cyan and orange bands. This does not show features in the $750-1050$ and 400-550 $\mathrm{nm}$ range, related to $e h_{22}$ and $e h_{33}$ of s-SWNTs, demonstrating removal of the majority of s-SWNTs, compared to the starting material (black dashed-dot line). Moreover, the absorption of the SC:SDS-1:4 dispersion (Figure $17 \mathrm{~d})$ has features associated with large diameter tubes $(1.4-1.5$ $\mathrm{nm})^{103}$ both for the aliquots extracted from the dark cyan and orange band. On the contrary, a larger diameter distribution is achieved for SC:SDS-4:1 (1.3-1.5 nm), Figure 17b. However, in both cases, there are no features corresponding to $\sim 1.0-1.2$ nm diameter. ${ }^{103}$ These results validate the assumption that the

\section{Emission wavelength (nm)}

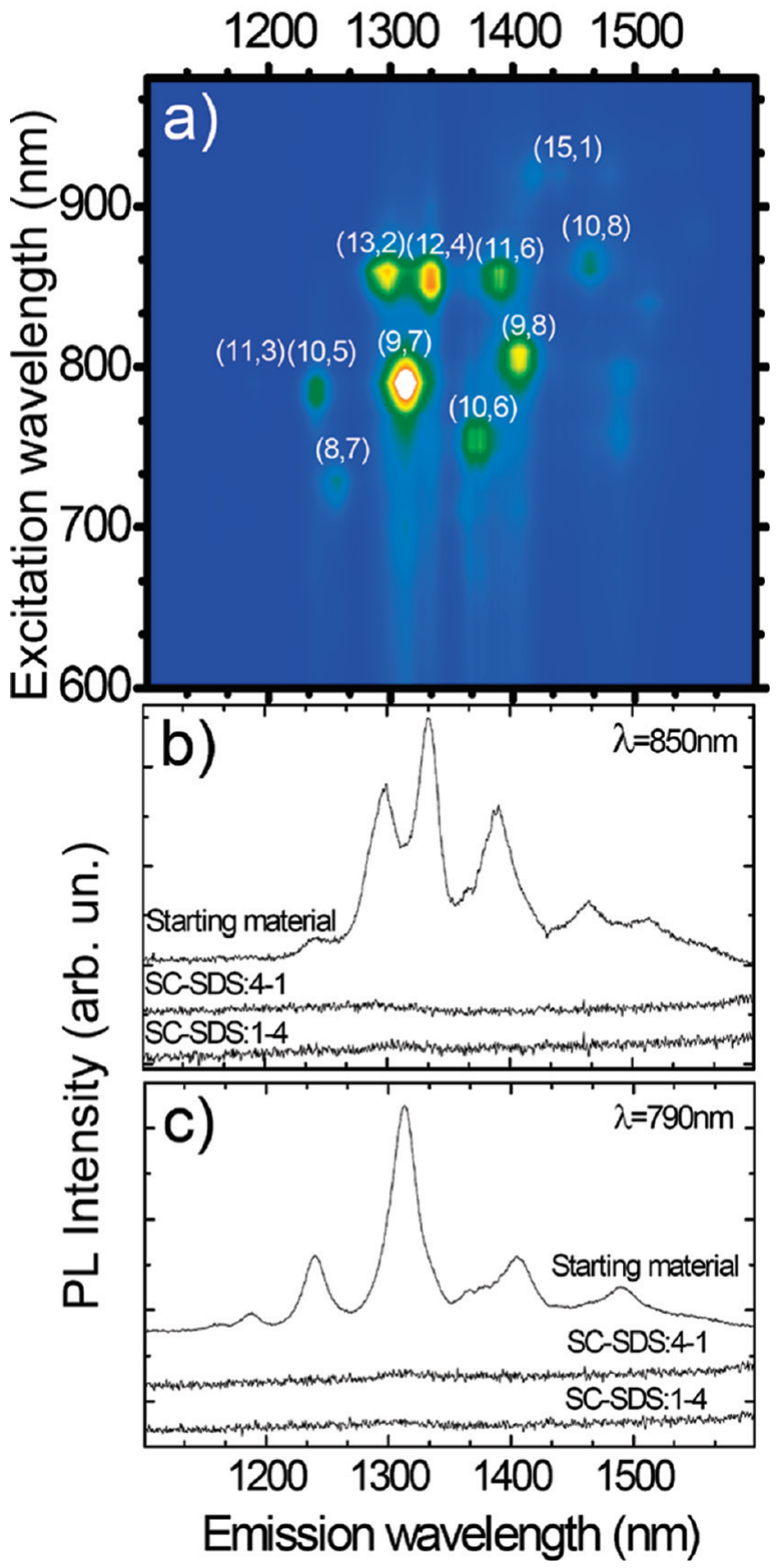

Figure 18. (a) PLE of LA SWNTs dispersed in SC. $(n, m)$ assigned following ref 103. (b and c) PL spectra of the SC:SDS-4:1 and SC: SDS-1:4 dispersions excited at (b) $790 \mathrm{~nm}$ and (c) $850 \mathrm{~nm}$. The spectra of the starting dispersion (SC) are also plotted.

activation energy for SC desorption from larger diameter tubes is lower than in smaller tubes. Thus, the change in buoyant density due to adsorption/desorption of surfactant molecules is more effective in larger tubes.

From OAS, we estimate $83 \%$ s- and $95 \% \mathrm{~m}-\mathrm{SWNTs}$ in the SC:SDS-4:1 and SC:SDS-1:4 samples, implying that m-separation is more effective. In particular, our m-separation is comparable to the best reported to date. ${ }^{37,48}$

Figure 18a plots the PLE map of LA SWNTs after the dispersion and individualization as for Section 2.2. The exciton-exciton resonances of $(8,7),(9,7),(9,8),(13,2),(12,4)$, $(15,1),(11,6),(10,5),(10,8),(11,3)$ are seen, corresponding to 

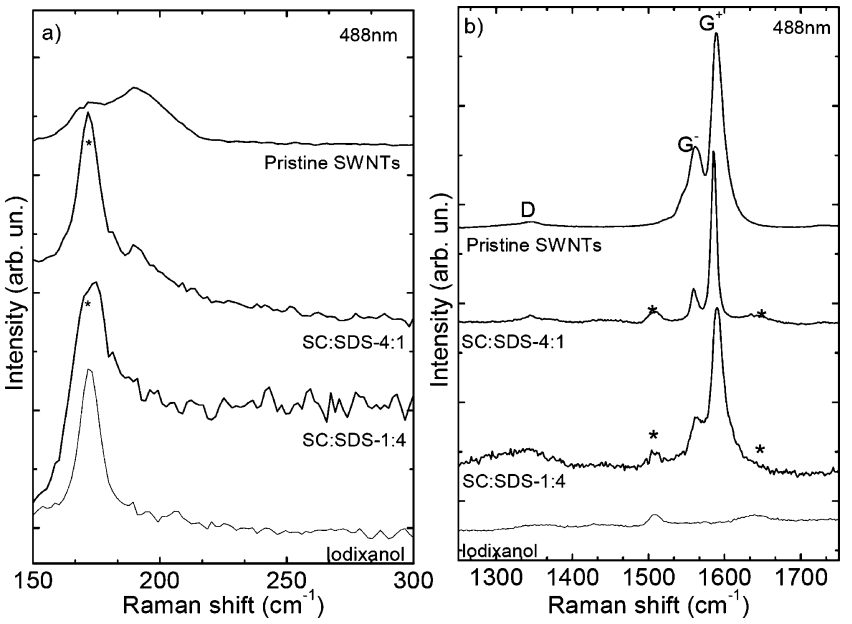

Figure 19. Raman spectra of LA SWNTs measured at $488 \mathrm{~nm}$. (a) $\mathrm{RBM}$ and (b) $\mathrm{G}$ region. Curves are for the pristine material and aliquots extracted in the orange band of the SC:SDS-4:1 sample and in the dark cyan band of the SC:SDS-1:4 one (see Figure 18). A reference iodixanol spectrum is also shown and marked *. Spectra are offset for clarity.

the $1.0-1.2 \mathrm{~nm}$ diameter range. On the contrary, the PL spectra of the aliquots extracted from the dark cyan and orange bands do not show any PL in the 1100-1600 nm range for excitation wavelengths of 790 and $850 \mathrm{~nm}$, respectively, Figures 18(b,c). For the aliquot extracted from the dark cyan band (SC:SDS-1: 4) this could be related to the removal of s-SWNTs. On the contrary, the aliquot extracted from the orange band (SC:SDS4:1) contains s-SWNTs such as $(13,9),(16,3),(16,6)$, seen in absorption, Figure 17(b). Their PL is not observed because their $e h_{11}$ emission $(1700-1800 \mathrm{~nm})$ is beyond our detection range.

Figures 19-22 plot the Raman spectra at 488, 514, 633, 785 $\mathrm{nm}$ for the aliquots extracted from the orange and dark cyan bands of the SC:SDS-4:1 and SC:SDS-1:4 dispersions. For comparison, the spectra of the raw material are also plotted. The latter shows RBMs in the range $150-230 \mathrm{~cm}^{-1}$. The RBM region in the enriched samples is dominated by the vibrational mode of iodine in Iodixanol at $169 \mathrm{~cm}^{-1} .{ }^{200}$ However, after separation, the peaks in the range $170-215 \mathrm{~cm}^{-1}$ are not detected at $488 \mathrm{~nm}$, Figure 19a. Only a peak at $190 \mathrm{~cm}^{-1}$ in the SC:SDS-4:1 sample is seen. The candidates for the assignment of this peak are the $(13,5)$ or $(14,3)$ RBMs. ${ }^{162,165}$ In Figure 19b the FWHM $\left(\mathrm{G}^{-}\right)$of the SC:SDS-1:4 sample is $\sim 60 \%$ larger with respect to that of SC:SDS-4:1. This is an indication of higher $\mathrm{m}-\mathrm{SWNTs}$ content. Doping could also modify the peaks' shapes, as discussed in section 2.4.3. ${ }^{178,179} \mathrm{We}$ do not expect doping to play a major role here for two reasons. First, both samples contain the same surfactants, even if with different concentration, so the doping contribution should be similar for both. Second, $\operatorname{Pos}\left(\mathrm{G}^{-}\right)$should upshift and become narrower as a consequence of either $\mathrm{p}$ or $\mathrm{n}$ doping. ${ }^{178,179}$

Figure 20 plots the Raman spectra at $514.5 \mathrm{~nm}$. The RBM region does not show any peaks due to the notch filter cut off at $\sim 230 \mathrm{~cm}^{-1}$, Figure 20a. The FWHM(G $\left(\mathrm{G}^{-}\right)$of the SC:SDS$1: 4$ sample is $\sim 20 \%$ larger with respect to the SC:SDS-4:1, which could indicate a higher percentage of $\mathrm{m}$ - tubes in the former fraction.

The spectra at $633 \mathrm{~nm}$, Figure 21a,b, are not significantly different. This is due to the resonant excitation of m-tubes at this wavelength, ${ }^{162,165}$ in agreement with the absorption of Figure 17, which shows residual m-tubes in the SC:SDS-4:1 samples. However, the spectrum of the SC:SDS-1:4 sample
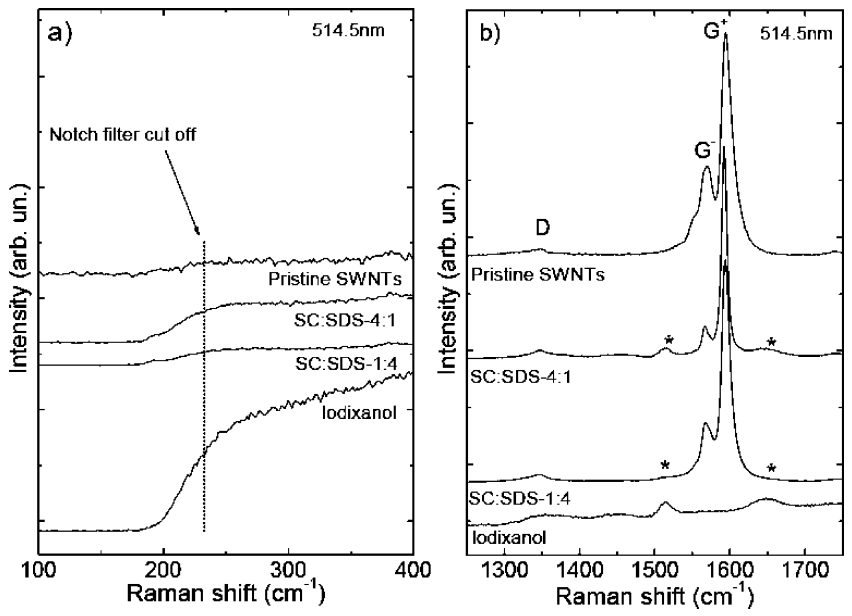

Figure 20. Raman spectra of LA SWNTs measured at $514.5 \mathrm{~nm}$. (a) $\mathrm{RBM}$ and (b) $\mathrm{G}$ region. The spectra for the raw material and the aliquots extracted in the orange band of the SC:SDS-4:1 and in the dark cyan band of the SC:SDS-1:4 dispersions, Figure 18a,c, are plotted. A reference iodixanol spectrum is also shown and marked *. Spectra are offset for clarity. Note that the RBM detection in (a) is limited by the filter cut off at $\sim 230 \mathrm{~cm}^{-1}$, indicated by the dotted line. Tubes with diameter $>1.0 \mathrm{~nm}$ cannot be detected.
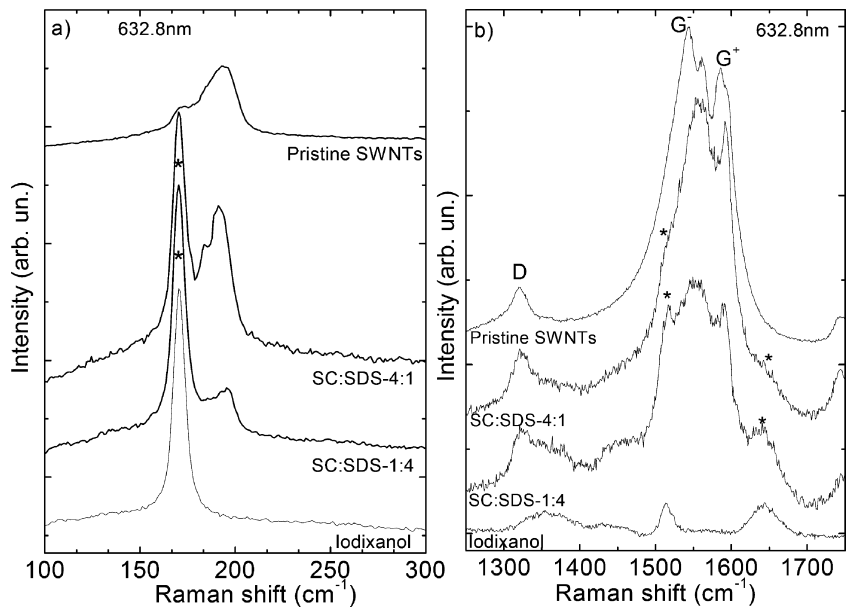

Figure 21. Raman spectra of LA SWNTs measured at $632.8 \mathrm{~nm}$. (a) RBM and (b) G region. The spectra for the pristine LA SWNT material and the aliquots extracted in the orange band of the SC:SDS-4:1 and in the dark cyan band of the SC:SDS-1:4, Figure 18a,c, are plotted. A reference iodixanol spectrum is also shown, marked with *. Spectra are offset for clarity.

has a $\mathrm{FWHM}\left(\mathrm{G}^{-}\right) \sim 10 \mathrm{~cm}^{-1}$ larger with respect to SC:SDS$4: 1$, which could be taken as indication of a higher amount of m-SWNTs.

A clearer evidence of $\mathrm{m} / \mathrm{s}$ separation is seen at $785 \mathrm{~nm}$. While the RBM region, Figure 22a, in the SC:SDS-4:1 sample does not show any peak (note that $(9,7)$ and $(10,5)$ should be in resonance at this excitation), ${ }^{103,165}$ the SC:SDS-1:4 sample has peaks at 156,158 , and $160 \mathrm{~cm}^{-1}$, due to $(17,5)$ and $(19,1)$ or $(18,3)$ and $(14,8)$, respectively, ${ }^{162,165}$ while the peaks in the $198-225 \mathrm{~cm}^{-1}$ region, observed in the starting material, disappear in both enriched fractions. The RBMs in resonance with this wavelength are those of $(9,8),(14,1),(9,7),(10,5)$, $(10,6),(12,4)$, and $(13,2){ }^{162,165}$ All of these are s-SWNTs and, in principle, should be detectable in SC:SDS-4:1. However, their average diameter is around $1.0-1.2 \mathrm{~nm}$ and not efficiently separated by DGU as demonstrated by the absorption and PLE measurements in Figures 17 and 18. $\mathrm{FWHM}\left(\mathrm{G}^{-}\right)$is broader in 

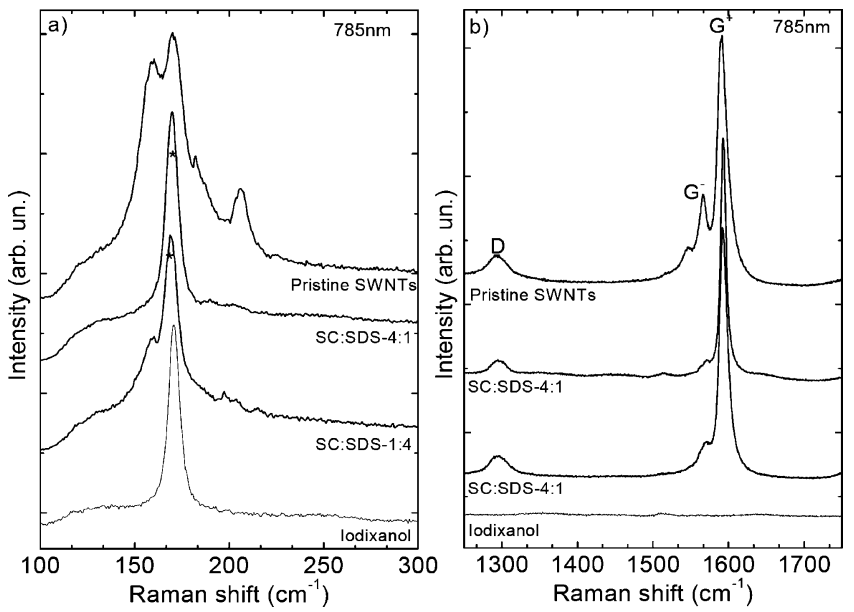

Figure 22. Raman spectra of LA SWNTs measured at $785 \mathrm{~nm}$. (a) RBM and (b) G region. The spectra for the pristine LA SWNT material and the aliquots extracted in the orange band of the SC:SDS-4:1 and in the dark cyan band of the SC:SDS-1:4, Figure 18a,c, are plotted. A reference iodixanol spectrum is also shown and marked*. Spectra are offset for clarity.

SC:SDS-1:4, with respect to both SC:SDS-4:1 and the starting material, fingerprint of m-separation, see Figure $22 \mathrm{~b}$.

Thus, the Raman results, in agreement with absorption and PLE, show that the $\mathrm{m} / \mathrm{s}$ separation is diameter selective and involves predominantly larger diameter tubes (1.3-1.5 $\mathrm{nm}$ range for the LA SWNTs used here).

3.4. Chirality Enrichment. Section 3.2 discussed the effectiveness of SC to get a very narrow diameter distribution (peaked at $\sim 0.75 \mathrm{~nm}$ ) for CoMoCAT SWNTs. However, as demonstrated by OAS, PLE and Raman measurements, in addition to the predominant $(6,5)$ species, the sample contains many other chiralities, Figure 12e,f, with both s-SWNTs, such as $(6,4),(9,1)$, and $(8,3)$, and $\mathrm{m}-\mathrm{SWNT}$ such as $(6,6)$ and $(7,4)$, in the diameter range $0.68-0.81 \mathrm{~nm}$. For example, the $(7,4)$, with diameter $0.75 \mathrm{~nm}$ and chiral angle $\theta=21^{\circ}$ is geometrically close to the $(6,5)\left(d=0.75 \mathrm{~nm}\right.$ and $\left.\theta=27^{\circ}\right)$. However, $(7,4)$ is $\mathrm{m}$ and $(6,5)$ is $\mathrm{s}$; hence, they can be separated due to their different electronic properties. ${ }^{37}$ Thus, in order to reduce the $(n, m)$ combinations and obtain the highest percentage of a single chirality, we carry out a two step DGU: a m/s separation exploiting a cosurfactant mixture (TDC-SDS) followed by diameter separation (SC), see section 2.3.3. We use the TDC/ SDS combination for the m- versus s-SWNTs separation of the CoMoCAT tubes because, from the diameter separation results in section 3.2, TDC was found to have higher affinity with s-SWNTs compared to m-SWNTs, as shown by the absorption spectrum in Figure 11b. We focus on $(6,5)$ enrichment, since its large band gap is very convenient to induce EET. ${ }^{26}$

Figure 23a is a photograph of the ultracentrifuge cell after the second DGU step. This shows a purple band and some residual sediment with higher buoyant density at the bottom. Absorption from the topmost fraction, red line in Figure 23(b), has strong bands at 572 and $983 \mathrm{~nm}$, associated with $e h_{22}$ and $e h_{11}$ of $(6,5) .{ }^{103}$ We estimate the absorption of $(6,5)$ to account for $\sim 78 \%$ of the total, considering the $e h_{11}$ of $\mathrm{m}-$ and $e h_{11}$ of s-SWNTs in the $400-1300 \mathrm{~nm}$ range, and $\sim 95 \%$ in the $800-1300 \mathrm{~nm}$ range, with respect to the $e h_{11}$ of s-tubes. Figure $23 \mathrm{~b}$ shows also the spectrum of the starting dispersion for comparison (black line).

The $(6,5)$ enrichment, along with the removal of s-SWNTs with similar diameter, such as $(8,3)$, is also evident in the PLE map of Figure 23c. This also shows another feature due to the

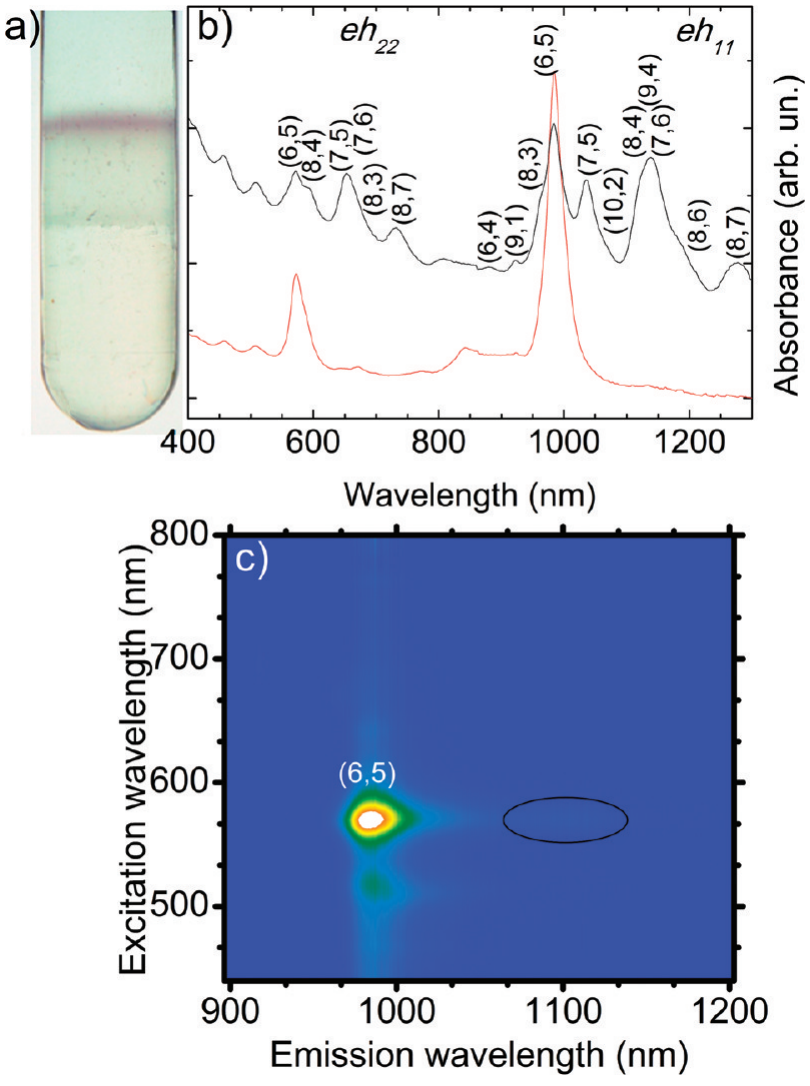

Figure 23. (a) Photograph and (b) absorption after a two steps enrichment of CoMoCAT SWNTs (red line). The spectrum of the starting TDC dispersion is plotted for comparison (black line). (c) PLE map of the enriched fraction. The ellipse indicates the $(6,5) e h_{11}-\mathrm{K}$ emission satellite. ${ }^{158}$ Chiralities are assigned following ref 103.
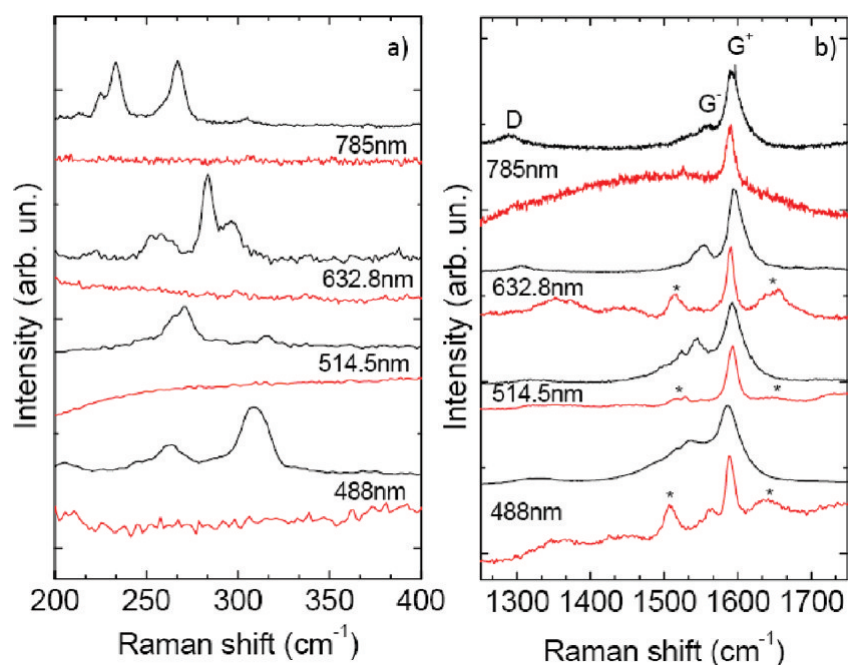

Figure 24. Raman spectra of CoMoCAT SWNTs measured at 488, 514,633 , and $785 \mathrm{~nm}$ (black lines) and after two steps enrichment (red lines) in the (a) RBM and (b) G peak region. The features marked * are Iodixanol. Spectra are offset for clarity.

$e h_{11}$-K emission satellite of $(6,5)$, at around $(570,1100 \mathrm{~nm})$ (see ref 158 and section 3.2.1).

Figure 24 compares the Raman spectra of pristine CoMoCAT powder (black lines) with the enriched sample (red lines). The pristine material shows RBMs related to m- and s-SWNTs. ${ }^{162}$ After DGU, the RBM region does not have detectable signal due to the removal of the majority of tubes, including $(8,3)$ and $(9,1)$. As discussed in section 3.2.1, the $(6,5)$ RBMs are not seen since we are off-resonance with respect to the $(6,5) e h_{22}{ }^{103}$ 
Figure 24b plots the $\mathrm{G}$ peak region. $\operatorname{Pos}\left(\mathrm{G}^{-}\right)$at $785 \mathrm{~nm}$ is downshifted $\left(13 \mathrm{~cm}^{-1}\right)$ with respect to the raw material, while $\operatorname{FWHM}\left(\mathrm{G}^{+}\right)$is $15 \mathrm{~cm}^{-1}$ narrower and $\operatorname{Pos}\left(\mathrm{G}^{+}\right)$is $4 \mathrm{~cm}^{-1}$ downshifted. Similar trends at 633,514 , and $488 \mathrm{~nm}$ confirm the presence in the enriched fraction of a narrow tube distribution with diameter $<0.8 \mathrm{~nm},{ }^{174}$ consistent with the $(6,5)$ diameter. The Raman spectra also confirm removal of the majority of other tubes, in agreement with absorption and PLE in Figure 23.

We note that ref 37 reported $\sim 84 \%$ enrichment of $(6,5)$ among s-SWNTs after three DGU iterations. DGU in a density constant medium with a cosurfactant mixture (SC-SDS) ${ }^{52}$ flavin mononucleotide ${ }^{54}$ and DNA ${ }^{55}$ resulted in enrichment of 68,85 , and $86 \%$ of a single chirality among s-SWNTs, respectively. DNA is highly selective toward s-SWNTs as also demonstrated by ref 56 , where chromatographic purification of up to $90 \%$ of a single-chirality was achieved. However, we need to consider the qualitative nature of these estimations. These are derived from different spectroscopic techniques such as Raman, ${ }^{177}$ PLE, ${ }^{52,54,55}$ or absorption. ${ }^{37,39,48,50,51}$ Moreover, even considering the same spectroscopic technique, different strategies for data extraction were used. Reference 201 reported significant discrepancies between transmission electron microscopy and spectroscopic results, both on chiral distribution and the $\mathrm{m} / \mathrm{s}$ ratios in enriched SWNTs.

Our results $(\sim 78 \%$ enrichment of single chirality over the totality of species and $\sim 95 \%$ among s-SWNTs) compare favorably the best data reported for all sorting techniques, confirming the viability of DGU for single chirality enrichment.

\section{Conclusions}

We tested different surfactants and polymers to sort SWNTs via DGU, focusing on the role of SWNT individualization and sidewall coverage. We got a narrow diameter distribution $(0.69-0.81 \mathrm{~nm})$ using SC in CoMoCAT tubes. Lower separation was achieved using SDC and TDC. We assigned the reduced selectivity of SDC and TDC to their different molecular structures, which determine a change in the formation of the micelles around the SWNTs sidewalls. We also showed that SDBS does not individualize SWNTs, and thus is ineffective for DGU. Separation in the range $0.84-0.91 \mathrm{~nm}$ was obtained using Pluronic F98. Using surfactants mixtures (SC-SDS) we separated $\mathrm{m} / \mathrm{s}-\mathrm{SWNTs}$ in the $1.3-1.5 \mathrm{~nm}$ range. Finally, combining diameter (using SC) and $\mathrm{m} / \mathrm{s}$ separation (using TDCSDS) we achieved 78\% (6,5) enrichment, removing most $\mathrm{m}-\mathrm{SWNTs}$ with similar diameter.

Acknowledgment. We are grateful to F. Hennrich for providing the LA tubes and to G. Calogero and S. A. Awan for useful discussions. We acknowledge funding from EPSRC grants G030480/1, H00274X/1, the ERC Grant NANOPOTS, a Royal Society Brian Mercer Award for Innovation, and International Joint Project. F.B. acknowledges funding from a Newton International Fellowship, T.H. from King's college, Cambridge, and P.H.T from Project No.G2009CB929300. A.C.F. is a Royal Society Wolfson Research Merit Award holder.

\section{References and Notes}

(1) Ding, L.; Tselev, A.; Wang, J.; Yuan, D.; Chu, H.; McNicholas, T. P.; Li, Y.; Liu, J. Nano Lett. 2009, 9, 800.

(2) Kim, W.; Choi, H. C.; Shim, M.; Li, Y.; Wang, D.; Dai, H. Nano Lett. 2002, 2, 703 .

(3) Li, Y. M.; Mann, D.; Rolandi, M.; Kim, W.; Ural, A.; Hung, S.; Javey, A.; Cao, J.; Wang, D. W.; Yenilmez, E.; Wang, Q.; Gibbons, J. F.; Nishi, Y.; Dai, H. Nano Lett. 2004, 4, 317.
(4) Qu, L.; Du, F.; Dai, L. Nano Lett. 2008, 8, 2682.

(5) Rao, C. N. R.; Voggu, R.; Govindaraj, A. Nanoscale. 2009, 1, 96.

(6) Harutyunyan, A. R.; Chen, G.; Paronyan, T. M.; Pigos, E. M.; Kuznetsov, O. A.; Hewaparakrama, K.; Kim, S. M.; Zakharov, D.; Stach, E. A.; Sumanasekera, G. U. Science 2009, 326, 116.

(7) Wang, B.; Poa, C. H. P.; Wei, L.; Li, L. J.; Yang, Y.; Chen, Y. J. Am. Chem. Soc. 2007, 129, 9014.

(8) Li, X.; Tu, X.; Zaric, S.; Welsher, K.; Seo, W. S.; Zhao, W.; Dai, H. J. Am. Chem. Soc. 2007, 129, 15770.

(9) Scardaci, V.; Sun, Z.; Wang, F.; Rozhin, A. G.; Hasan, T.; Hennrich, F.; White, I. H.; Milne, W. I.; Ferrari, A. C. Adv. Mater. 2008, 20,4040 .

(10) Sun, Z.; Rozhin, A. G.; Wang, F.; Scardaci, V.; Milne, W. I.; White, I. H.; Hennrich, F.; Ferrari, A. C. Appl. Phys. Lett. 2008, 93, 061114.

(11) Wang, F.; Rozhin, A. G.; Scardaci, V.; Sun, Z.; Hennrich, F.;

White, I. H.; Milne, W. I.; Ferrari, A. C. Nat. Nano. 2008, 3, 738.

(12) Hasan, T.; Sun, Z.; Wang, F.; Bonaccorso, F.; Tan, P. H.; Rozhin, A. G.; Ferrari, A. C. Adv. Mater. 2009, 21, 3874.

(13) Rozhin, A. G.; Scardaci, V.; Wang, F.; Hennrich, F.; White, I. H.; Milne, W. I.; Ferrari, A. C. Phys. Status Solidi B 2006, 243, 3551.

(14) Sun, Z.; Rozhin, A. G.; Wang, F.; Hasan, T.; Popa, D.; O'Neil, W.; Ferrari, A. C. Appl. Phys. Lett. 2009, 95, 253102.

(15) Bonaccorso, F.; Sun, Z.; Hasan, T.; Ferrari, A. C. Nature Photon 2010, 4, 611 .

(16) Srivastava, A.; Srivastava, O. N.; Talapatra, S.; Vajtal, R.; Ajayan, P. M. Nat. Mater. 2004, 3, 610 .

(17) Green, A. A.; Hersam, M. C. Nano Lett. 2008, 8, 1417.

(18) Marago, O. M.; Gucciardi, P. G.; Bonaccorso, F.; Calogero, G.; Scardaci, V.; Rozhin, A. G.; Ferrari, A. C.; Jones, P. H.; Saija, R.; Borghese, F.; Denti, P.; Iati, M. A. Physica E 2008, 40, 2347.

(19) Jones, P. H.; Palmisano, F.; Bonaccorso, F.; Gucciardi, P. G.; Calogero, G.; Ferrari, A. C.; Marago, O. M. ACS Nano 2009, 3, 3077.

(20) Marago, O. M.; Jones, P. H.; Bonaccorso, F.; Scardaci, V.; Gucciardi, P. G.; Rozhin, A. G.; Ferrari, A. C. Nano Lett. 2008, 8, 3211.

(21) Trushkevych, O.; Collings, N.; Hasan, T.; Scardaci, V.; Ferrari, A. C.; Wilkinson, T. D.; Crossland, W. A.; Milne, W. I.; Geng, J.; Johnson,

B. F. G.; Macaulay, S. J. Phys. D: Appl. Phys. 2008, 41, 125106.

(22) Khoo, I. C.; Ding, J.; Zhang, Y.; Chen, K.; Diaz, A. Appl. Phys. Lett. 2003, 82, 3587.

(23) Lee, W.; Chiu, C. S. Opt. Lett. 2001, 26, 521.

(24) Huang, C. Y.; Hu, C. Y.; Pan, H. C.; Lo, K. Y. Jpn. J. Appl. Phys. 2005, 44, 8077.

(25) Chen, H. Y.; Lee, W. Appl. Phys. Lett. 2006, 88, 222105.

(26) Tan, P. H.; Rozhin, A. G.; Hasan, T.; Hu, P.; Scardaci, V.; Milne,

W. I.; Ferrari, A. C. Phys. Rev. Lett. 2007, 99, 137402.

(27) Tan, P. H.; Hasan, T.; Bonaccorso, F.; Scardaci, V.; Rozhin, A. G.;

Milne, W. I.; Ferrari, A. C. Physica E 2008, 40, 2352.

(28) Hong, Z. R.; Lessmann, R.; Maennig, B.; Huang, Q.; Harada, K.; Riede, M.; Leo, K. J. Appl. Phys. 2009, 106, 064511.

(29) Duesberg, G. S.; Muster, J.; Krstic, V.; Burghard, M.; Roth, S. Appl. Phys. A: Mater. Sci. Process. 1998, 67, 117.

(30) Arnold, K.; Hennrich, F.; Krupke, R.; Lebedkin, S.; Kappes, M. M. Phys. Status Solidi B 2006, 243, 3073.

(31) Heller, D. A.; Mayrhofer, R. M.; Baik, S.; Grinkova, Y. V.; Usrey, M. L.; Strano, M. S. J. Am. Chem. Soc. 2004, 126, 14567.

(32) Vetcher, A. A.; Srinivasan, S.; Vetcher, I. A.; Abramov, S. M.; Kozlov, M.; Baughman, R. H.; Levene, S. D. Nanotechnology 2006, $174263-4269$.

(33) Zheng, M.; Jagota, A.; Strano, M. S.; Santos, A. P.; Barone, P.; Chou, S. G.; Diner, B. A.; Dresselhaus, M. S.; McLean, R. S.; Onoa, G. B.; Samsonidze, G. G.; Semke, E. D.; Usrey, M.; Walls, D. J. Science 2003, $302,1545$.

(34) Strano, M. S.; Zheng, M.; Jagota, A.; Onoa, G. B.; Heller, D. A.; Barone, P. W.; Usrey, M. L. Nano Lett. 2004, 4, 543.

(35) Doorn, S. K.; Strano, M. S.; O'Connell, M. J.; Haroz, E. H.; Rialon, K. L.; Hauge, R. H.; Smalley, R. E. J. Phys. Chem. B 2003, 10, 7-6063. 713.

(36) Arnold, M. S.; Stupp, S. I.; Hersam, M. C. Nano Lett. 2005, 5,

(37) Arnold, M. S.; Green, A. A.; Hulvat, J. F.; Stupp, S. I.; Hersam, M. C. Nat. Nano. 2006, $1,60$.

(38) Green, A. A.; Hersam, M. C. Mater. Today 2007, 10, 59

(39) Crochet, J.; Clemens, M.; Hertel, T. J. Am. Chem. Soc. 2007, 129, 8058 .

(40) Hennrich, F.; Arnold, K.; Lebedkin, S.; Quintillá, A.; Wenzel, W.; Kappes, M. Phys. Status Solidi B 2007, 244, 3896.

(41) Nair, N.; Kim, W. J.; Braatz, R. D.; Strano, M. S. Langmuir 2008, 24, 1790 .

(42) Krupke, R.; Hennrich, F.; Lohneysen, H.; Kappes, M. M. Science 2003, 301, 344.

(43) Krupke, R.; Hennrich, F.; Weber, H. B.; Kappes, M. M.; Lohneysen, H. V. Nano Lett. 2003, 3, 1019. 
(44) Krupke, R.; Hennrich, F.; Kappes, M. M.; Lohneysen, H. V. Nano Lett. 2004, 4, 1395 .

(45) Krupke, R.; Linden, S.; Rapp, M.; Hennrich, F. Adv. Mater. 2006, 18,1468 . 1571 .

(46) Kim, W. J.; Usrey, M. L.; Strano, M. S. Chem. Mater. 2007, 19,

(47) Tanaka, T.; Jin, H.; Miyata, Y.; Fujii, S.; Suga, H.; Naitoh, Y.; Minari, T.; Miyadera, T.; Tsukagoshi, K.; Kataura, H. Nano Lett. 2009, 9 , 1497. 034003

(48) Yanagi, K.; Miyata, Y.; Kataura, H. Appl. Phys. Express 2008, 1,

(49) Tanaka, T.; Jin, H.; Miyata, Y.; Kataura, H. Appl. Phys. Express 2008, 1, 114001 .

(50) Yanagi, K.; Iitsuka, T.; Fujii, S.; Kataura, H. J. Phys. Chem. C 2008, 112, 18889.

(51) Moshammer, K.; Hennrich, F.; Kappes, M. M. Nano Res. 2009, 2,599

(52) Wei, L.; Wang, B.; Goh, T. H.; Li, L. J.; Yang, Y. H.; Chan-

Park, M. B.; Chen, Y. J. Phys. Chem. B 2008, 112, 2771.

(53) Zheng, M.; Semke, E. D. J. Am. Chem. Soc. 2007, 129, 6084.

(54) Ju, S. Y.; Doll, J.; Sharma, I.; Papadimitrakopoulos, F. Nat. Nano. 2008, 3, 356

(55) Kim, S. N.; Kuang, Z.; Grote, J. G.; Farmer, B. L.; Naik, R. R. Nano Lett. 2008, 8, 4415. 250

(56) Tu, X.; Manohar, S.; Jagota, A.; Zheng, M. Nature 2009, 460,

(57) O’Connell, M. J.; Bachilo, S. M.; Huffman, C. B.; Moore, V. C.; Strano, M. S.; Haroz, E. H.; Rialon, K. L.; Boul, P. J.; Noon, W. H.; Kittrell C.; Ma, J.; Hauge, R. H.; Weisman, R. B.; Smalley, R. E. Science 2002 $297,593$.

(58) Moore, V. C.; Strano, M. S.; Haroz, E. H.; Hauge, R. H.; Smalley, R. E. Nano Lett. 2003, 3, 1379.

(59) Islam, M. F.; Rojas, E.; Bergey, D. M.; Johnson, A. T.; Yodh, A. G. Nano Lett. 2003, 3, 269.

(60) Haggenmueller, R.; Rahatekar, S. S.; Fagan, J. A.; Chun, J. H.; Becker, M. L.; Naik, R. R.; Krauss, T.; Carlson, L.; Kadla, J. F.; Trulove, P. C.; Fox, D. F.; DeLong, H. C.; Fang, Z. C.; Kelley, S. O.; Gilman, J. W. Langmuir 2008, 24, 5070 .

(61) O’Connell, M. J.; Boul, P.; Ericson, L. M.; Huffman, C.; Wang, Y.; Haroz, E.; Kuper, C.; Tour, J.; Ausman, K. D.; Smalley, R. E. Chem Phys. Lett. 2001, 342, 265.

(62) Minami, N.; Kim, Y.; Miyashita, K.; Kazaoui, S.; Nalini, B. Appl. Phys. Lett. 2006, 88, 093123.

(63) Hasan, T.; Scardaci, V.; Tan, P. H.; Rozhin, A. G.; Milne, W. I.; Ferrari, A. C. J. Phys. Chem. C 2007, 111, 12594.

(64) Hasan, T.; Tan, P. H.; Bonaccorso, F.; Rozhin, A.; Scardaci, V.; Milne, W.; Ferrari, A. C. J. Phys. Chem. C 2008, 112, 20227.

(65) Zheng, M.; Jagota, A.; Semke, E. D.; Diner, B. A.; Mclean, R. S.;

Lustig, S. R.; Richardson, R. E.; Tassi, N. G. Nat. Mater. 2003, 2, 338.

(66) Ausman, K. D.; Piner, R.; Lourie, O.; Ruoff, R. S.; Korobov, M.

J. Phys. Chem. B 2000, 104, 8911.

(67) Krupke, R.; Hennrich, F.; Hampe, O.; Kappes, M. M. J.Phys. Chem. B 2003, 107, 5667

(68) Giordani, S.; Bergin, S. D.; Nicolosi, V.; Lebedkin, S.; Kappes, M. M.; Blau, W. J.; Coleman, J. N. J. Phys. Chem. B 2006, 110, 15708 (69) Bergin, S. D.; Nicolosi, V.; Streich, P. V.; Giordani, S.; Sun, Z.; Windle, A. H.; Ryan, P.; Niraj, N. P. P.; Wang, Z.-T., T.; Carpenter, L.;

Blau, W. J.; Boland, J. J.; Hamilton, J. P.; Coleman, J. N. Adv. Mater. 2008, 20, 1876

(70) Landi, B. J.; Ruf, H J.; Worman, J. J.; Raffaelle, R. P. J.Phys. Chem. B 2004, 108, 17089.

(71) Hasan, T.; Scardaci, V.; Tan, P. H.; Rozhin, A. G.; Milne, W. I.; Ferrari, A. C. Physica E 2008, 40, 2414.

(72) Scardaci, V.; Rozhin, A. G.; Tan, P. H.; Wang, F.; White, I. H.; Milne, I. W.; Ferrari, A. C. Physica Status Solidi B 2007, 244, 4303.

(73) Nish, A.; Hwang, J.-Y.; Doig, J.; Nicholas, R. J. Nat. Nano. 2007, 2,640

(74) Peng, X; Komatsu, N.; Bhattacharya, S. Shimawaki, T; Aonuma, S.; Kimura, T.; Osuka, A. Nat. Nano. 2007, 2, 361.

(75) Peng, X.; Komatsu, N. S.; Kimura, T.; Osuka, A. J. Am. Chem. Soc. 2007, 129, 15947 69.

(76) Green, A. A.; Duch, M. C.; Hersam, M. C. Nano Res. 2009, 2,

(77) Ghosh, S.; Bachilo, S. M.; Weisman, R. B. Nat. Nano. 2010, 5 ,

(78) Kim, S. N.; Luo, Z.; Papadimitrakopoulos, F. Nano Lett. 2005, 5, 2500.

(79) Strano, M. S.; Dyke, C. A.; Usrey, M. L.; Barone, P. W.; Allen, M. J.; Shan, H.; Kittrell, C.; Hauge, R. H.; Tour, J. M.; Smalley, R. E. Science 2003, 301, 1519.

(80) Strano, M. S. J. Am. Chem. Soc. 2003, 125, 16148.

(81) Toyoda, S.; Yamaguchi, Y.; Hiwatashi, M.; Tomonari, Y.; Murakami, H.; Nakashima, N. Chem. Asian J. 2007, 2, 145.
(82) Chen, F. M.; Wang, B.; Chen, Y.; Li, L. J. Nano Lett. 2007, 7, 3013

(83) Tromp, R. M.; Afzali, A.; Freitag, M.; Mitzi, D. B.; Chen, Z. Nano Lett. 2008, 8, 469 .

(84) Ahuja, S. In Handbook of bioseparations; Ahuja, S., Ed.; Academic Press: San Diego, CA, 2000; p 1.

(85) O’Keefe, D. O. In Handbook of bioseparations; Ahuja, S., Ed.; Academic Press: San Diego, CA, 2000; p 23.

(86) Westermeier, R. Electrophoresis in Practice; Wiley-VCH Verlag GmbH \& Co.: Weinheim, Germany, 2005.

(87) Landers, J. P. In Handbook of Capillary and Microchip Electrophoresis and Associated Microtechniques; Landers, J. P., Ed.; CRC Press: Boca Raton, FL, 2008.

(88) Brakke, M. K. Arch. Biochem. 1953, 45, 275.

(89) Brakke, M. K.; Daly, J. M. Science 1965, 148, 387.

(90) Svedberg, T.; Pedersen, K. O. The Ultracentrifuge 1940.

(91) Hersam, M. Nat. Nano. 2008, 3, 387.

(92) Bahr, J. L.; Tour, J. M. J. Mater. Chem. 2002, 12, 1952.

(93) Singh, P.; Campidelli, S.; Giordani, S.; Bonifazi, D.; Bianco, A.; Prato, M. Chem. Soc. Rev. 2009, 38, 2214.

(94) Hersam, M. C. Nature 2009, 460, 186.

(95) Doorn, S. K.; Fields, R. E.; Hu, H.; Hamon, M. A.; Haddon, R. C.; Selegue, J. P.; Majidi, V. J. Am. Chem. Soc. 2002, 124, 3169.

(96) Vijayaraghavan, A.; Sciascia, C.; Dehm, S.; Lombardo, A.; Bonetti, A.; Ferrari, A. C.; Krupke, R. ACS Nano 2009, 3, 1729.

(97) Essig, S.; Marquardt, C. W.; Vijayaraghavan, A.; Ganzhorn, M.; Dehm, S.; Hennrich, F.; Ou, F.; Green, A. A.; Sciascia, C.; Bonaccorso, F.; Bohnen, K. P.; Lohneysen, H. v.; Kappes, M. M.; Ajayan, P. M.; Hersam, M. C.; Ferrari, A. C.; Krupke, R. Nano Lett. 2010, 10, 1589.

(98) Shin, D. H.; Kim, J.-E.; Shim, H. C.; Song, J.-W.; Yoon, J.-H.; Kim, J.; Jeong, S.; Kang, J.; Baik, S.; Han, C.-S. Nano Lett. 2008, 8, 4380. (99) Maeda, Y.; Kimura, S.; Kanda, M.; Hirashima, Y.; Hasegawa, T.; Wakahara, T.; Lian, Y. F.; Nakahodo, T.; Tsuchiya, T.; Akasaka, T.; Lu, J.; Zhang, X. W.; Gao, Z. X.; Yu, Y. P.; Nagase, S.; Kazaoui, S.; Minami, N.; Shimizu, T.; Tokumoto, H.; Saito, R. J. Am. Chem. Soc. 2005, 127, 10287.

(100) Chen, Z. H.; Du, X.; Du, M. H.; Rancken, C. D.; Cheng, H. P.; Rinzler, A. G. Nano Lett. 2003, 3, 1245.

(101) Williams, J. W.; Holde, K. E.; Baldwin, R. L.; Fujita, H. Chem. Rev. 1958, 58, 715 .

(102) Ifft, J. B ; Vinograd, J. J. Phys. Chem 1966, 70, 2814

(103) Weisman, R. B.; Bachilo, S. M. Nano Lett. 2003, 3, 1235.

(104) Fagan, J. A.; Becker, M. L.; Chun, J.; Nie, P.; Bauer, B. J.; Simpson, J. R.; Hight-Walker, A.; Hobbie, E. K. Langmuir 2008, 24, 13880. (105) Kitiyanan, B.; Alvarez, W. E.; Harwell, J. H.; Resasco, D. E. Chem. Phys. Lett. 2000, 317, 497.

(106) Resasco, D. E.; Alvarez, W. E.; Pompeo, F.; Balzano, L.; Herrera,

J. E.; Kitiyanan, B.; Borgna, A. J. Nanopart. Res. 2002, 4, 131.

(107) Lebedkin, S.; Schweiss, P.; Renker, B.; Malik, S.; Hennrich, F.; Neumaier, M.; Stoermer, C.; Kappes, M. M. Carbon 2002, 40, 40417.

(108) Lebedkin, S.; Hennrich, F.; Skipa, T.; Kappes, M. M. J. Phys.

Chem. B 2003, 107, 1945 .

(109) Wenseleers, W.; Vlasov, I.; Goovaerts, E.; Obraztsova, E.; Lobach, A.; Bouwen, A. Adv. Funct. Mater. 2004, 14, 1105.

(110) Duro, R.; Souto, C.; Gomez-Amoza, J. L.; Martinez-Pacheco, R.; Concheiro, A. Drug Dev. Ind. Pharm. 1999, 25, 817.

(111) Romanini, D.; Avalle, G.; Farruggia, B.; Nerli, B.; Pico, G. Chem. Biol. Interact. 1998, 115, 247.

(112) Richard, C.; Balavoine, F.; Schultz, P.; Ebbesen, T. W.; Mioskowski, C. Science 2003, 300, 775.

(113) Yuan, S. L.; Cai, Z. T.; Xu, G. Y.; Jiang, Y. S. Colloid Polym. Sci. 2002, 280, 630.

(114) Calafato, N. R.; Pico, G. Colloid Surf. B 2006, 47, 198

(115) Small, D. M.; Penkett, S. A.; Chapman, D. Biochem. Biophys. Acta 1969, 176, 178.

(116) Mazer, N. A.; Carey, M. C.; Kwasnick, R. F.; Benedek, G. B.

Biochemistry 1979, 18, 3064 .

(117) An iso-osmolar contrasting agent.

(118) Bildirici, L.; Rickwood, D. J. Immunol. Methods 2000, 240, 93.

(119) Patel, D.; Rickwood, D. J. Immunol. Methods 1995, 184, 71.

(120) Kaminsky, M. Discuss. Faraday Soc. 1957, 24, 171.

(121) Collins, K.; Washabaugh, M. Q. Rev. Biophys. 1985, 18, 323.

(122) Cacace, M. G.; Landau, E. M.; Ramsden, J. J. Q. Rev. Biophys.

1997, 30, 241

(123) Coombs, D. H.; Watts, N. R. M. Anal. Biochem. 1985, 148, 254.

(124) Zhang, J.; Kang, D. E.; Xia, W.; Okochi, M.; Mori, H.; Selkoe,

D. J.; Koo, E. H. J. Biol. Chem. 1998, 273, 12436.

(125) Smale, G.; Sasse, J. Anal. Biochem. 1992, 203, 352

(126) Vieira, O. V.; Laranjinha, J. A. N.; Madeira, V. M. C.; Almeida,

L. M. J. Lipid Res. 1996, 37, 2715.

(127) Cohen, R.; Claverie, J. M. Biopolymers 1975, 14, 1701.

(128) Garber, R. C.; Yoder, O. C. Anal. Biochem. 1983, 135, 416.

(129) Charlwood, P. A. Br. Med. Bull. 1966, 22, 121. 
(130) Crochet, J.; Clemens, M.; Hertel, T. Phys. Status Solidi B 2007, 244,3964

(131) Stillemark, P.; Boren, J.; Andersson, M.; Larsson, T.; Rustaeus,

S.; Karlsson, K. A.; Olofsson, S. O. J. Biol. Chem. 2000, 275, 10506.

(132) Brakke, M. K. Anal. Biochem. 1963, 5, 271.

(133) Brakke, M. K. Advan. Virus Res. 1960, 7, 193.

(134) Szybalski, W. Experientia 1960, 16, 164.

(135) Tan, K. B. Anal. Biochem. 1972, 45, 306.

(136) Britten, R. J.; Roberts, R. B. Science 1960, 131, 32

(137) Allington, R. W.; Brakke, M. K.; Nelson, J. W.; Aron, C. G.;

Larkins, B. A. Anal. Biochem. 1976, 73, 78.

(138) Kozinsky, B.; Marzari, N. Phys. Rev. Lett. 2006, 96, 4.

(139) Pfeffer, W. Osmotic Investigations: Studies on Cell Mechanics;

Van Nostrand Reinhold Co.: New York, 1985.

(140) Guell, D. C.; Brenner, H. Ind. Eng. Chem. Res. 1996, 35, 3004. (141) Niyogi, S.; Boukhalfa, S.; Chikkannanavar, S. B.; Mcdonald, T. J.;

Heben, M. J.; Doorn, S. K. J. Am. Chem. Soc. 2007, 129, 1898.

(142) Hagen, A.; Hertel, T. Nano Lett. 2003, 3, 383.

(143) Heller, D. A.; Barone, P. W.; Swanson, J. P.; Mayrhofer, R. M.; Strano, M. S. J. Phys. Chem. B 2004, 108, 6905.

(144) Reich, S.; Thomsen, C.; Ordejón, P. Phys. Rev. B 2002, 65, 155411

(145) Wang, F.; Sfeir, M. Y.; Huang, L.; Huang, X. M. H.; Wu, Y.;

Kim, J.; Hone, J.; O’Brien, S.; Brus, L. E.; Heinz, T. F. Phys. Rev. Lett. 2006, 96, 167401.

(146) O'Connell, M. J.; Sivaram, S.; Doorn, S. K. Phys. Rev. B 2004, $69,235415$.

(147) Walsh, A. G.; Vamivakas, A. N.; Yin, Y.; Ünlü, M. S.; Goldberg,

B. B.; Swan, A. K.; Cronin, S. B. Nano Lett. 2007, 7, 1485.

(148) Lefebvre, J.; Fraser, J. M.; Homma, Y.; Finnie, P. Appl. Phys. A:

Mater. Sci. Process. 2004, 78, 1107.

(149) Ando, T. J. Phys. Soc. Jpn. 2004, 73, 3351.

(150) Perebeinos, V.; Tersoff, J.; Avouris, P. Phys. Rev. Lett. 2004, 92, 257402

(151) Workman, J. J. In Applied Spectroscopy: A Compact Reference for Practitioners; Workman, J. J., Springsteen, A., Eds.; Academic Press: San Diego, 1998.

(152) Maeda, Y.; Kimura, S. I.; Hirashima, Y.; Kanda, M.; Lian, Y.; Wakahara, T.; Akasaka, T.; Hasegawa, T.; Tokumoto, H.; Shimizu, T.;

Kataura, H.; Miyauchi, Y.; Maruyama, S.; Kobayashi, K.; Nagase, S. J. Phys. Chem. B 2004, 108, 18395.

(153) McDonald, T. J.; Blackburn, J. L.; Metzger, W. K.; Rumbles, G.; Heben, M. J. J. Phys. Chem. C 2007, 111, 17894.

(154) Perebeinos, V.; Tersoff, J.; Avouris, P. Phys. Rev. Lett. 2005, 94, 027402 .

(155) Plentz, F.; Ribeiro, H. B.; Jorio, A.; Strano, M. S.; Pimenta, M. A.

Phys. Rev. Lett. 2005, 95, 247401.

(156) Lefebvre, J.; Finnie, P. Phys. Rev. Lett. 2007, 98, 167406.

(157) Kato, T.; Hatakeyama, R. J. Am. Chem. Soc. 2008, 130, 8101.

(158) Torrens, O. N.; Zheng, M.; Kikkawa, J. M. Phys. Rev. Lett. 2008,

$101,157401$.

(159) Qian, H.; Georgi, C.; Anderson, N.; Green, A. A.; Hersam, M. C.;

Novotny, L.; Hartschuh, A. Nano Lett. 2008, 8, 1363.

(160) Reich, S.; Thomsen, C.; Robertson, J. Phys. Rev. Lett. 2005, 95 , 077402 .

(161) Rao, A. M.; Richter, E.; Bandow, S.; Chase, B.; Eklund, P. C.; Williams, K. A.; Fang, S.; Subbaswamy, K. R.; Menon, M.; Thess, A.;

Smalley, R. E.; Dresselhaus, G.; Dresselhaus, M. S. Science 1997, 275, 187.

(162) Telg, H.; Maultzsch, J.; Reich, S.; Hennrich, F.; Thomsen, C. Phys. Rev. Lett. 2004, 93, 177401.

(163) Meyer, J. C.; Paillet, M.; Michel, T.; Moreac, A.; Neumann, A.; Duesberg, G. S.; Roth, S.; Sauvajol, J. L. Phys. Rev. Lett. 2005, 95, 4.

(164) Fantini, C.; Jorio, A.; Souza, M.; Strano, M. S.; Dresselhaus, M. S.;

Pimenta, M. A. Phys. Rev. Lett. 2004, 93, 147406.

(165) Kataura, H.; Kumazawa, Y.; Maniwa, Y.; Umezu, I.; Suzuki, S.;

Ohtsuka, Y.; Achiba, Y. Synth. Met. 1999, 103, 2555.

(166) Paillet, M.; Michel, T.; Meyer, J. C.; Popov, V. N.; Henrard, L.;

Roth, S.; Sauvajol, J. L. Phys. Rev. Lett. 2006, 96, 257401.
(167) Jorio, A.; Saito, R.; Hafner, J. H.; Lieber, C. M.; Hunter, M.; McClure, T.; Dresselhaus, G.; Dresselhaus, M. S. Phys. Rev. Lett. 2001, 86, 1118.

(168) Araujo, P. T.; Doorn, S. K.; Kilina, S.; Tretiak, S.; Einarsson, E.; Maruyama, S.; Chacham, H.; Pimenta, M. A.; Jorio, A. Phys. Rev. Lett. 2007, 98, 067401.

(169) Araujo, P. T.; Jorio, A.; Dresselhaus, M. S.; Sato, K.; Saito, R. Phys. Rev. Lett. 2009, 103, 146802.

(170) Ferrari, A. C.; Robertson, J. Phys. Rev. B 2000, 61, 14095.

(171) Ferrari, A. C.; Robertson, J. Phys. Rev. B 2001, 64, 075414.

(172) Ferrari, A. C. Solid State Commun. 2007, 143, 47.

(173) Lazzeri, M.; Piscanec, S.; Mauri, F.; Ferrari, A. C.; Robertson, J. Phys. Rev. B 2006, 73, 155426 .

(174) Piscanec, S.; Lazzeri, M.; Robertson, J.; Ferrari, A. C.; Mauri, F. Phys. Rev. B 2007, 75, 035427.

(175) Ferrari, A. C.; Meyer, J. C.; Scardaci, V.; Casiraghi, C.; Lazzeri, M.; Mauri, F.; Piscanec, S.; Jiang, D.; Novoselov, K. S.; Roth, S.; Geim, A. K. Phys. Rev. Lett. 2006, 97, 187401.

(176) Mohiuddin, T. M. G.; Lombardo, A.; Nair, R. R.; Bonetti, A.; Savini, G.; Jalil, R.; Bonini, N.; Basko, D. M.; Galiotis, C.; Marzari, N.; Novoselov, K. S.; Geim, A. K.; Ferrari, A. C. Phys. Rev. B 2009, 79, 205433.

(177) Jorio, A.; Souza Filho, A. G.; Dresselhaus, G.; Dresselhaus, M. S.; Swan, A. K.; Ünlü, M. S.; Goldberg, B. B.; Pimenta, M. A.; Hafner, J. H.; Lieber, C. M.; Saito, R. Phys. Rev. B 2002, 65, 155412.

(178) Das, A.; Sood, A. K.; Govindaraj, A.; Saitta, A. M.; Lazzeri, M.;

Mauri, F.; Rao, C. N. R. Phys. Rev. Lett. 2007, 99, 136803.

(179) Tsang, J. C.; Freitag, M.; Perebeinos, V.; Liu, J.; Avouris, Ph. Nat. Nano. 2007, 2, 725.

(180) Das, A.; Pisana, S.; Piscanec, S.; Chakraborty, B.; Saha, S. K.;

Waghmare, U. V.; Yang, R.; Krishnamurhthy, H. R.; Geim, A. K.; Ferrari,

A. C.; Sood, A. K. Nat Nano. 2008, 3, 210.

(181) Jorio, A.; Santos, A. P.; Ribeiro, H. B.; Fantini, C.; Souza, M.;

Vieira, J. P. M.; Furtado, C. A.; Jiang, J.; Saito, R.; Balzano, L.; Resasco,

D. E.; Pimenta, M. A. Phys. Rev. B 2005, 72, 075207.

(182) Tanford, C. Proc. Natl. Acad. Sci. U.S.A. 1979, 76, 4175.

(183) Tanford, C. The hydrophobic effect; Wiley: New York, 1973.

(184) Chari, K.; Frank, C. W.; Gast, A. Langmuir 1989, 5, 1096.

(185) Eskilsson, K.; Tiberg, F. Macromolecules 1997, 30, 6323.

(186) Israelachvili, J. Intermolecular and surface force, 2nd ed.; Academic Press: New York, 1991.

(187) Roda, A.; Hofmann, A. F.; Mysels, K. J. J. Biol. Chem. 1983, 258,6362 .

(188) Yurekli, K.; Mitchell, C. A.; Krishnamoorti, R. J. Am. Chem. Soc.

2004, 126, 9902

(189) Bachilo, S. M.; Strano, M. S.; Kittrell, C.; Hauge, R. H.; Smalley,

R. E.; Weisman, R. B. Science 2002, 298, 2361.

(190) Piscanec, S.; Lazzeri, M.; Mauri, F.; Ferrari, A. C. Eur. Phys. J. 2007, 148, 159.

(191) Woodford, F. P. J. Lipid Res. 1969, 10, 539.

(192) Ekwall, P.; Fontell, K.; Sten, A. Proc. Int. Congr. Surf. Act., 2nd

1957, 1, 357.

(193) Cohen, R. J.; Benedek, G. B. J. Mol. Biol. 1976, 108, 151.

(194) Tanford, C. J. Phys. Chem. 1974, 78, 2469.

(195) Nagarajan, R.; Ruckenstein, E. Langmuir 1991, 7, 2934.

(196) Lu, J.; Nagase, S.; Zhang, X. W.; Wang, D.; Ni, M.; Maeda, Y.;

Wakahara, T.; Nakahodo, T.; Tsuchiya, T.; Akasaka, T.; Gao, Z. X.; Yu,

D. P.; Ye, H. Q.; Mei, W. N.; Zhou, Y. S. J. Am. Chem. Soc. 2006, 128, 5114 .

(197) Jost, O.; Gorbunov, A. A.; Pompe, W.; Pichler, T.; Friedlein, R.; Knupfer, M.; Reibold, M.; Bauer, H. D.; Dunsch, L.; Golden, M. S. J. Appl. Phys. Lett. 1999, 75, 2217.

(198) Umlong, I. M.; Ismail, K. Colloid Surf. A: Physicochem. Eng. Aspects 2007, 299, 8.

(199) Chen, J. M.; Su, T. M.; Mou, C. Y. J. Phys. Chem. 1986, 90, 2418.

(200) Priebe, H.; Dustag, H.; Gacek, M.; Hagen, E.; Homestad, O. M.; Larsen, Å.; Sjøgren, C. E.; Thomassen, T. Acta Radiol. 1995, 36, 21.

(201) Sato, Y.; Yanagi, K.; Miyata, Y.; Suenaga, K.; Kataura, H.; Iijima, S. Nano Lett. 2008, 8, 3151.

JP1030174 